\title{
Inverse Problem and Estimates for Periodic Zakharov-Shabat systems
}

\author{
Evgeni Korotyaev* \\ Universität Potsdam, Institut für Mathematik \\ e-mail: evgeni@math.uni-potsdam.de
}

March 17, 2000

\begin{abstract}
Consider the Zakharov-Shabat (or Dirac) operator $T_{z s}$ on $L^{2}(\mathbb{R}) \oplus L^{2}(\mathbb{R})$ with real periodic vector potential $q=\left(q_{1}, q_{2}\right) \in H=L^{2}(\mathbb{T}) \oplus L^{2}(\mathbb{T})$. The spectrum of $T_{z s}$ is absolutely continuous and consists of intervals separated by gaps $\left(z_{n}^{-}, z_{n}^{+}\right), n \in \mathbb{Z}$. ¿From the Dirichlet eigenvalues $m_{n}, n \in \mathbb{Z}$ of the Zakharov-Shabat equation with Dirichlet boundary conditions at 0,1 , the center of the gap and the square of the gap length we construct the gap length mapping $g: H \rightarrow \ell^{2} \oplus \ell^{2}$. Using nonlinear functional analysis in Hilbert spaces, we show that this mapping is a real analytic isomorphism. Our proof relies on new identities and estimates contained in the second part of the our paper.
\end{abstract}

\section{Introduction and main results}

Consider the Zakharov-Shabat (or Dirac) operator $T_{z s}$ acting on $L^{2}(\mathbb{R}) \oplus L^{2}(\mathbb{R})$ and having the form

$$
T_{z s}=J \frac{d}{d x}+V(x), \quad V(x) \equiv\left(\begin{array}{cc}
q_{1}(x) & q_{2}(x) \\
q_{2}(x) & -q_{1}(x)
\end{array}\right) \quad J \equiv\left(\begin{array}{cc}
0 & 1 \\
-1 & 0
\end{array}\right),
$$

where $q=\left(\begin{array}{l}q_{1} \\ q_{2}\end{array}\right) \in H=L^{2}(\mathbb{R} / \mathbb{Z}) \oplus L^{2}(\mathbb{R} / \mathbb{Z})$, is a real 1-periodic vector function of $x \in \mathbb{R}$. The spectrum of $T_{z s}$ is purely absolutely continuous and is given by the set $\cup \sigma_{n}$, with spectral band $\sigma_{n}=\left[z_{n-1}^{+}, z_{n}^{-}\right]$. These intervals are separated by gaps $\gamma_{n}=\left(z_{n}^{-}, z_{n}^{+}\right)$with

*Partially supported by Russian Fund of Fundamental Research, INTAS and SFB 288. Permanent address: Math. Dept. 2, ETU, 5 Prof. Popov Str., St.Petersburg, 197376 Russia 
length $\left|\gamma_{n}\right| \geqslant 0$. If a gap $\gamma_{n}$ is degenerate, i.e. $\gamma_{n}=\emptyset$, then the corresponding spectral bands $\sigma_{n}, \sigma_{n+1}$ merge. We use the Zakharov-Shabat (or Dirac) equation for a vector -function $f(x)$

$$
J f^{\prime}+V f=z f, \quad z \in \mathbb{C}, \quad f(x)=\left(\begin{array}{l}
f_{1}(x) \\
f_{2}(x)
\end{array}\right),
$$

where $f_{1}, f_{2}$ are functions in $x \in \mathbb{R}$. Here and below $\left({ }^{\prime}\right)=\partial / \partial x,\left({ }^{\circ}\right)=\partial / \partial z, \partial / \partial q=$ $\left(\partial_{1}, \partial_{2}\right)=\left(\partial / \partial q_{1}, \partial / \partial q_{2}\right)$. We recall well known results on the 1-dimensional ZakharovShabat operator (see $[\mathrm{Kr}],[\mathrm{LS}]$ for details). The boundary value problem (1.1) with the condition $f(0)=f(1)$ is called periodic and the boundary value problem (1.1) with the condition $f(0)=-f(1)$ is called antiperiodic. We denote the eigenvalues of the periodic problem by $z_{2 n}^{ \pm}$and the eigenvalues of the anti-periodic problem by $z_{2 n+1}^{ \pm}, n \in \mathbb{Z}$. These form the periodic and anti periodic spectrum. It is well known that $\ldots<z_{2 n-1}^{-} \leqslant z_{2 n-1}^{+}<z_{2 n}^{-} \leqslant$ $z_{2 n}^{+}<\ldots$, and $z_{n}^{ \pm}=n(\pi+o(1))$, as $\quad|n| \rightarrow \infty$. We consider the following $2 \times 2$-matrix valued fundamental solution $\psi=\psi(x, z, q)$ :

$$
J \psi^{\prime}+V \psi=z \psi, \quad \psi(0, z, q)=I, \quad z \in \mathbb{C}, q \in H_{C} .
$$

Rewrite this $2 \times 2$ - matrix valued solution $\psi$ in terms of the vector-functions $\vartheta, \varphi$ :

$$
\psi=\left(\begin{array}{ll}
\vartheta_{1} & \varphi_{1} \\
\vartheta_{2} & \varphi_{2}
\end{array}\right), \quad \vartheta(x, z, q)=\left(\begin{array}{c}
\vartheta_{1}(x, z, q) \\
\vartheta_{2}(x, z, q)
\end{array}\right), \quad \varphi(x, z, q)=\left(\begin{array}{l}
\varphi_{1}(x, z, q) \\
\varphi_{2}(x, z, q)
\end{array}\right) .
$$

Introduce the Lyapunov function $\Delta(z, q)=\frac{1}{2} \operatorname{Tr} \psi(1, z, q)$. Note that $\Delta\left(z_{n}^{ \pm}, q\right)=(-1)^{n}, n \in$ $\mathbb{Z}$, and the function $\dot{\Delta}(z, q)$ has a zero $z_{n}$ in a "closed gap" $\left[z_{n}^{-}, z_{n}^{+}\right]$. There exists the unique conformal mapping (the quasimomentum) $k: \mathcal{Z} \rightarrow \mathcal{K}$ such that (see [Mi1])

$$
\begin{gathered}
\cos k(z)=\Delta(z, q), \quad z \in \mathcal{Z}=\mathbb{C} \backslash \cup \bar{\gamma}_{n}, \quad \mathcal{K}=\mathbb{C} \backslash \cup,_{n}, \quad{ }_{n}=\left(\pi n-i h_{n}, \pi n+i h_{n}\right) \\
k(z)=z-\frac{\|q\|^{2}+o(1)}{2 z}, \quad z \rightarrow i \infty, \quad \text { and } \quad k(z)=z+o(1), \quad z \rightarrow \pm \infty,
\end{gathered}
$$

where, ${ }_{n}$ is the excised vertical slit, the height $h_{n} \geqslant 0$ is defined by the equation $\cosh h_{n}=$ $(-1)^{n} \Delta\left(z_{n}, q\right) \geqslant 1$. We emphasize that the introduction of the quasimomentum $k(z)$ provides a natural labeling of all gaps $\gamma_{n}$ (including the empty ones!) by demanding that $k(\cdot)$ maps the gap $\gamma_{0}$ on the vertical slit, $0=\left(-i h_{0}, i h_{0}\right)$. This determination of a fixed reference point will be of crucial important later on. We introduce the Hilbert spaces

$$
\ell_{m}^{2}=\left\{f=\left\{f_{n}\right\}_{\mathbb{Z}}, \quad\|f\|^{2}+\|f\|_{m}^{2}<\infty\right\}, \quad\|f\|_{m}^{2} \equiv \sum(2 \pi n)^{2 m}\left|f_{n}\right|^{2}<\infty, \quad m \geqslant 1,
$$

and $\ell_{0}^{2}=\ell^{2}=\left\{f=\left\{f_{n}\right\}_{\mathbb{Z}}, \quad\|f\|^{2} \equiv \sum\left|f_{n}\right|^{2}<\infty\right\}$. We consider the "Dirichlet problem" for the Dirac operator, i.e. the problem (1.1) with the condition $f_{1}(0, z)=f_{1}(1, z)=0$. Let $m_{n}(q)$ denote the Dirichlet eigenvalue, it is well known that $m_{n}(q) \in\left[z_{n}^{-}, z_{n}^{+}\right]$. Define the mapping $g: q \rightarrow g(q)=\left\{g_{n}(q)\right\}_{n \in \mathbb{Z}}$ from $H$ into $\ell^{2} \oplus \ell^{2}$ by the rule: $g_{n}=\left(g_{n 1}, g_{n 2}\right) \in \mathbb{R}^{2}$, where the length is defined by the equation $\left|g_{n}(q)\right|=\left|\gamma_{n}\right| / 2$ and the components have the form:

$$
g_{n 1}=\frac{1}{2}\left(z_{n}^{+}+z_{n}^{-}\right)-m_{n}(q), \quad g_{n 2}=\left.|| g_{n}\right|^{2}-\left.g_{n 1}^{2}\right|^{1 / 2} \operatorname{sign} h_{n 2}, \quad h_{n 2}=-\log \left|\varphi_{2}\left(1, m_{n}(q), q\right)\right| .
$$


The "physical" interpretation of $g_{n 1}, g_{n 2}$ has the same sense as for the Hill operator, see [K1] for details. We formulate the main result of the present paper.

Theorem 1.1. The mapping $g: H \rightarrow \ell^{2} \oplus \ell^{2}$ is a real analytic isomorphism and the following estimates are fulfilled:

$$
\frac{1}{\sqrt{2}}\|g\| \leqslant\|q\| \leqslant 2\|g\|(1+\|g\|) .
$$

Remarks. i) From the value $g(q)$ we can compute the gap length $\left|\gamma_{n}\right|$, sign $g_{n 2}$, and $g_{n 1}$ for any $n \in \mathbb{Z}$. However, we do not know the position of the gaps and the Dirichlet eigenvalues. Arbitrarily fixing the position of some gap, in general, corresponds to a non-periodic potential. ii) The Gelfand-Levitan-Marchenko equation and a trace formula are not used in the proof. iii) Estimates (1.3) were proved in [K3].

Theorem 1.1 generalizes the result of [K1] to the case of the Dirac operator with periodic potential. This generalization requires essentially different proofs, since the technique that was used for studying the scalar Hill operator could not be directly generalized to the matrix Dirac operator. This induced us to reconsider the original version of the proof (Theorem A), this led us evidently to discover simplifications, and also improvements in the estimates. The proof presented here depends on new identities (see Theorem 1.2), which seem to us also to have independent interest. They are analogs of corresponding identities for the Hill operator which were first proved in [K5]. We also need new estimates (see Theorem 1.3).

Let $k=u+i v, z=x+i y$. We introduce the moments

$Q_{n}=\frac{1}{\pi} \int x^{n} v(x) d x, \quad S=\frac{1}{\pi} \int u(x) x v(x) d x, \quad U=\frac{1}{\pi} \int v(x)^{3} d x, \quad P_{n}=\frac{1}{\pi} \int u(x)^{n} v(x) d x$,

$n \geqslant 0$. Here and below an integral with no limits as indicated above denotes the integration over $\mathbb{R}^{1}$ or $\mathbb{R}^{2}$. We remark that, when we consider the functions $v(x)$ on the real line we take the functions $v(x)=v(x+i 0)$.

Consider the defocussing cubic non-linear Schrödinger equation (NLS)

$$
-J \frac{d \psi}{d t}=-\psi_{x x}+2|\psi|^{2} \psi, \quad \psi=\psi(t, x)=\left(\begin{array}{c}
\psi_{1}(t, x) \\
\psi_{2}(t, x)
\end{array}\right), \quad \psi(0, x)=q(x) .
$$

It is well known that the NLS is a completely integrable infinite dimensional Hamiltonian system. The periodic spectrum of (1.1) is invariant under the flow of NLS. We have the following identities for the Dirac operator

$$
\mathcal{H}_{0}(q) \equiv \frac{1}{2}\|q\|^{2} \equiv \int_{0}^{1}|q(t)|^{2} d t=Q_{0}=\frac{1}{2 \pi} \iint\left|z^{\prime}(k)-1\right|^{2} d u d v,
$$

where the first one was proved in [Mi1] and the second one was proved in [KK1], and

$$
\mathcal{H}_{1}(q) \equiv \frac{1}{2}\left(J q^{\prime}, q\right)=2 Q_{1}, \quad \mathcal{H}_{2}(q) \equiv \frac{1}{2} \int_{0}^{1}\left(\left|q^{\prime}\right|^{2}+|q|^{4}\right) d t=4 Q_{2}
$$

Here $\mathcal{H}_{0}$ is particle number, $\mathcal{H}_{1}$ is momentum, $\mathcal{H}_{2}$ is energy (or the Hamiltonian). We now formulate the main new identities used in our approach. 
Theorem 1.2. Let $q, q^{\prime} \in H$. Then the following identities are fulfilled:

$$
\begin{gathered}
\frac{1}{\pi} \iint\left|\left(z^{2}(k)-k^{2}\right)^{\prime}\right|^{2} d u d v=8 S, \\
\frac{1}{4}\left(J q^{\prime}, q\right)=Q_{1}=P_{1}, \\
\frac{1}{8} \int_{0}^{1}\left(\left|q^{\prime}\right|^{2}+|q|^{4}\right) d t=Q_{2}=S+\frac{Q_{0}^{2}}{2}=P_{2}-\frac{1}{3} U+Q_{0}^{2},
\end{gathered}
$$

in particular,

$$
S+\frac{1}{3} U=P_{2}+\frac{Q_{0}^{2}}{2} .
$$

Let us introduce the sequences $\gamma=\left\{\left|\gamma_{n}\right|\right\}, h=\left\{h_{n}\right\}, A=\left\{A_{n}\right\}, a=\left\{a_{n}\right\}$, where $a_{n}=$ $\left|A_{n}\right|^{1 / 2}$, and $A_{n}=(1 / \pi) \int_{\gamma_{n}} v(x) d x \geqslant 0, \quad n \in \mathbb{Z}$, is the action variable for the NLS (see $[\mathrm{FM}])$. Note that $\mathcal{H}_{0}(q)=\sum A_{n}$. In fact, due to Theorem 1.2 we rewrite $\mathcal{H}_{1}$ and the main part of $\mathcal{H}_{2}$ in terms of the action variables:

$$
\mathcal{H}_{1}(q)=\sum(2 \pi n) A_{n}, \quad \mathcal{H}_{2}(q)=\frac{1}{4} \sum(2 \pi n)^{2} A_{n}+\mathcal{H}_{0}^{2}(q)-\frac{1}{3} U .
$$

Note that the Hamiltonian $\mathcal{H}_{2}$, the number of particles $\mathcal{H}_{0}$ and the momentum $\mathcal{H}_{1}$ depend only on the action-variables $A_{n}, n \in \mathbb{Z}$. Introduce the frequencies $\omega_{n}=\frac{\partial H}{\partial A_{n}}, n \in \mathbb{Z}$. The parameters $\omega_{n}$ are very important since the angle variables are $\phi_{n}(t)=\omega_{n} t+\phi_{n}(0), t \geqslant$ $0, n \in \mathbb{Z}$. Identity (1.8) yields $\omega_{n}=(\pi n)^{2}+2 \mathcal{H}_{0}-\frac{1}{3} \frac{\partial U}{\partial A_{n}}$. Here, $(\pi n)^{2}$ is the main term in $\omega_{n}$ as $|n| \rightarrow \infty$. We now formulate our main new estimates.

Theorem 1.3. Let $q, q^{\prime} \in H$. Then the following estimates are fulfilled:

$$
\begin{gathered}
h_{+}^{2} \leqslant 2 Q_{0} \leqslant 2 \sqrt{2 Q_{2}}, \quad Q_{0} \leqslant\left(\left|\gamma_{0}\right|+\|\gamma\|_{1}\right)^{2}, \quad h_{+}=\sup h_{n}, \\
Q_{2} \leqslant \frac{1}{\pi 2}\|h\|_{1}^{2}+\frac{4}{\pi^{2}}\|h\|^{4}, \quad\|h\|_{1}^{2} \leqslant(2 \pi)^{3} \beta^{2} Q_{2}, \quad \beta=\max \left\{1, \frac{h_{+}}{\pi}\right\} \\
\frac{1}{4}\|a\|_{1}^{2}+\frac{1}{3}\|a\|^{4} \leqslant Q_{2} \leqslant \frac{1}{4}\|a\|_{1}^{2}+\|a\|^{4}, \\
\frac{1}{32}\|\gamma\|^{2}+\frac{1}{192}\|\gamma\|_{1}^{2} \leqslant Q_{2} \leqslant \pi \beta^{2}\|\gamma\|_{1}^{2}+4 Q_{0}^{2} .
\end{gathered}
$$

Remark that such estimates for $q \in H$ were obtained in [K3]. The norms $f_{0}^{2}+\|f\|_{1}^{2}$ and $\|f\|^{2}+\|f\|_{1}^{2}$ are equivalent. Theorem 1.3 gives the double-sided estimates of $\left|\gamma_{0}\right|^{2}+\|\gamma\|_{1}^{2}$ (the appropriately normed total gap length), $h_{0}^{2}+\|h\|_{1}^{2}$ (the total height of vertical slits), $a_{0}^{2}+\|a\|_{1}^{2}$, (the "total" action variable) and the energy $\mathcal{H}_{2}$.

There are various methods of solving inverse problems. We shortly describe the "direct approach" from [GT2], [KK1], based on nonlinear functional analysis. Suppose that $H, H_{1}$ are real separable Hilbert spaces. The derivative of a map $f: H \rightarrow H_{1}$ at a point $y \in H$ is a bounded linear map from $H$ into $H_{1}$, which we denote by $d_{y} f$. A map $f: H \rightarrow H_{1}$ is compact on $H$, if it maps a weakly convergent sequence in $H$ into a strongly convergent 
sequence in $H_{1}$. A map $f: H \rightarrow H_{1}$ is a real analytic isomorphism between $H$ and $H_{1}$, if $f$ is bijective and both $f$ and $f^{-1}$ are real analytic maps. Let $H_{C}$ be the complexification of the real Hilbert space $H$. We formulate the key result of the direct method, proved in [KK1], with the needed modification in [K8].

Theorem A. Let $H, H_{1}$ be real separable Hilbert spaces with norms $\|\cdot\|,\|\cdot\|_{1}$. Suppose that a map $f: H \rightarrow H_{1}$ satisfies the following conditions:

i) $f$ is real analytic,

ii) the operator $d_{q} f$ has an inverse for all $q \in H$,

iii) there is a nondecreasing function $\xi:[0, \infty) \rightarrow[0, \infty), \xi(0)=0$, such that $\|q\| \leqslant$ $\xi\left(\|f(q)\|_{1}\right)$ for all $q \in H$

iv) there exists a basis $\left\{e_{n}\right\}_{n \in \mathbb{Z}}$ of $H_{1}$ such that each map $\left(f(\cdot), e_{n}\right)_{1}: H \rightarrow \mathbb{R}, n \in \mathbb{Z}$, is compact,

v) for each $\xi>0$ the set $\left\{q: \sum n^{2}\left(f(q), e_{n}\right)_{1}^{2}<\xi\right\}$ is compact.

Then $f$ is a real analytic isomorphism between $H$ and $H_{1}$.

A great numbers of papers are devoted to the inverse problem for the 1-dimension differential operator with periodic coefficients. Marchenko and Ostrovski [MO1-2] proved the continuous isomorphism of the vertical slit mapping for the Hill operator. Garnett and Trubowitz [GT1] proved that both of the vertical slit mapping and the gap length mapping are real analytic isomorphisms for the case of even potentials. Kargaev and the author [KK1] reproved the result of Garnet and Trubowitz [GT1] by the direct method. Moreover, they considered other mappings. Note that the proofs by the direct method are short but this approach needs some estimates (see iii) in Theorem A and (1.3)). The author [K2] reproved the result of [MO1-2] by the direct method. In order to get the estimates we need the "global quasimomentum" which was introduced into the spectral theory of the Hill operator by Firsova [F], [F1] and by Marchenko-Ostrovski [MO1] simultaneously.

Note that the main results of Firsova was devoted to the inverse problem for the operator $F y=-y^{\prime \prime}+p(x) y+V(x) y$ in $L^{2}(\mathbb{R})$ where $p$ is a fixed 1-periodic real potential and $V$ is decreasing real one. She generalized the well known result of Faddeev [Fa] (the case $p=0$ ) for the operator $F$ with the fixed $p \neq 0$, but unfortunately she repeated his error (see [DT]). Then the inverse problem for the operator $F$ is open.

Double-sided estimates for various parameters of the Hill operator (the norm of a periodic potential, effective masses, gap lengths, height of slits and so on ) were obtained in [K2-4, 7] and for the Dirac operator in [KK2], [K2], [K5]. Pöschel and Trubowitz [PT] wrote a book concerning the inverse Dirichlet problem. Krein $[\mathrm{Kr}]$ obtained various results about the Lyapunov function for the Dirac operator. In [Mi1-2] Misura extent the results of [MO12] for the periodic Dirac operator. In [BGGK] the authors proved that the "gap length mapping" for the Dirac operator (for the case of $q, q^{\prime} \in H$ ) is a real analytic isomorphism. We feel, however, that there is a gap in the proof of real analyticity in [Ka] (analyticity seems only to have been proved for each single component of the gap length mapping), and this proof was referred to in the subsequent work on the Dirac operator. Futhermore, it seems to us unclear how in [BGGK] the gaps are labeled without using the quasimomentum. Our approach avoids all these difficulties, by using the quasimomentum, a different gap length mapping and providing all necessary asymptotic estimates. 
We finish the introduction by briefly explaining how the proof by a direct approach will go, i.e., how we verify conditions i)-v) of Theorem A for the mapping $g$. Remark that the theorem from [KK1], does not apply since there exists a big difference between the asymptotics of gap lengths for the Hill operator and for the Dirac operator (see (1.9)). Asymptotics for the Hill operator is more convenient than asymptotics for the Dirac operator. Note that in the proof we only use some results from [K1], [K9-10], [PT] and the estimates (1.3) from [K8] (see also [K3]). The checking of $\mathrm{i}$ ) is based on the analyticity of the functions $\psi(\cdot, z, q), \Delta(z, q)$ of $z \in \mathbb{C}, q \in H_{C}$. In order to obtain the analyticity of the mapping $g$ we need to prove the real analyticity of the mappings $z_{n}^{-}(\cdot)+z_{n}^{+}(\cdot),\left|g_{n}(\cdot)\right|^{2}, m_{n}(\cdot)$. To check ii), we prove that the Fréchet derivatives of the map is a Fredholm operator of index zero with zero-dimensional kernels and, therefore, is invertible. Here we use a result of Paley-Wiener about entire functions (see Lemma 4.3) and the following fact: for any fixed $q \in H$ the vectors $\left\{\left(d_{q} h_{c n}\right),\left(d_{q} m_{n}\right), n \in \mathbb{Z}\right\}$ form a basis of $H$ (see [K9]). We emphasize the important role of the entire function $\partial \Delta(z, q)$. The verification of iii) is based on estimates (1.3) proved in [K3]. To check iv), we use the compactness of the mappings $z_{n}^{ \pm}(\cdot): H \rightarrow \mathbb{R}, m_{n}(\cdot): H \rightarrow \mathbb{R}$ and the fundamental solutions. The checking of $\mathrm{v}$ ) is based on estimates given in Theorem 1.3 .

\section{Preliminaries}

Below we need the simple identities for $J, V, J_{1}=\left(\begin{array}{cc}1 & 0 \\ 0 & -1\end{array}\right), J_{2}=\left(\begin{array}{cc}0 & 1 \\ 1 & 0\end{array}\right)$ :

$$
\begin{gathered}
J^{2}=-I, \quad J_{1} J_{2}=J, \quad J J_{1}=-J_{2}, \quad J J_{2}=J_{1}, \\
e^{z J}=I \cos z+J \sin z, \quad z \in \mathbb{C}, \\
J V=-V J, \quad V=q_{1} J_{1}+q_{2} J_{2},
\end{gathered}
$$

and identities (2.2-3) yield

$$
e^{z J} V=V e^{-z J}, \quad z \in \mathbb{C} .
$$

The $2 \times 2$ - matrix valued solution of the following equation

$$
J y^{\prime}-z y=f, \quad y(0, z, q)=I, \quad z \in \mathbb{C}, f \in H_{C},
$$

has the form

$$
y(x, z)=e^{-z x J}-\int_{0}^{x} e^{-z J(x-t)} J f(t) d t .
$$

Then the solution of Eq. (1.2) has the representation

$$
\psi(x, z, q)=e^{-z x J}+\int_{0}^{x} e^{-z J(x-t)} J V(t) \psi(t, z, q) d t .
$$

We construct the solution of this integral equation. It is clear that Eq. (2.7) has the solution as a power series in $q$. That is

$$
\psi(x, z, q)=\sum_{n \geqslant 0} \psi_{n}(x, z, q)
$$


where the functions $\psi_{n}$ are defined by the relations:

$$
\begin{aligned}
& \psi_{0}(x, z)=\psi_{0}(x, z, q)=e^{-z x J}=\left(\begin{array}{cc}
\cos z x & -\sin z x \\
\sin z x & \cos z x
\end{array}\right), \\
& \psi_{n}(x, z, q)=\int_{0}^{x} e^{-z J(x-t)} J V(t) \psi_{n-1}(t, z, q) d t, \quad n \geqslant 1,
\end{aligned}
$$

and here for fixed $x, z$, the function $\psi_{n}$ is a multi-linear form on $H_{C} \times \ldots \times H_{C}$. Using $(2.10)$ we have

$$
\psi_{1}(x, z, q)=\int_{0}^{x} e^{-z J(x-t)} J V(t) e^{-z t J} d t=\int_{0}^{x} e^{-z J(x-2 t)} J V(t) d t,
$$

and then

$$
\begin{gathered}
\psi_{1}=-\int_{0}^{x}\left(\begin{array}{cc}
q_{1}(t) \sin \xi-q_{2}(t) \cos \xi & q_{1}(t) \cos \xi+q_{2}(t) \sin \xi \\
q_{1}(t) \cos \xi+q_{2}(t) \sin \xi & -q_{1}(t) \sin \xi+q_{2}(t) \cos \xi
\end{array}\right) d t, \quad \xi=z(2 t-x), \\
\psi_{2}=\int_{0}^{x} e^{-z J(x-t)} J V(t) \psi_{1}(t, z, q) d t=\int_{0}^{x} d t_{1} \int_{0}^{t_{1}} e^{-z J\left(x-2 t_{1}+2 t_{2}\right)} V\left(t_{1}\right) V\left(t_{2}\right) d t_{2} .
\end{gathered}
$$

Proceeding by induction,

$$
\psi_{n}(x, z, q)=\int_{0}^{x} d t_{1} \int_{0}^{t_{1}} d t_{2} \ldots \int_{0}^{t_{n}} e^{-z J\left(x-t_{1}+2 t_{2} \ldots+(-1)^{n} 2 t_{n}\right)} J V\left(t_{1}\right) \ldots J V\left(t_{n}\right) d t_{n}
$$

and

$$
\begin{gathered}
\psi_{2 n}(x, z, q)=\int_{0}^{x} d t_{1} \ldots \int_{0}^{t_{2} n} e^{-z J\left(x-t_{1}+2 t_{2} \ldots+2 t_{2 n}\right)} V\left(t_{1}\right) \ldots V\left(t_{2 n}\right) d t_{2 n}, \\
\psi_{2 n+1}(x, z, q)=\int_{0}^{x} d t_{1} \ldots \int_{0}^{t_{2} n+1} e^{-z J\left(x-t_{1}+2 t_{2} \ldots-2 t_{2 n+1}\right)} J V\left(t_{1}\right) \ldots V\left(t_{2 n+1}\right) d t_{2 n+1} .
\end{gathered}
$$

We consider the following $2 \times 2$ - matrix valued fundamental solution $\Psi=\Psi(x, z, q, t)$ with the parameter $t$ :

$$
J \Psi^{\prime}+V(x+t) \Psi=z \Psi, \quad \Psi(0, z, q, t)=I, \quad t \in \mathbb{R}, z \in \mathbb{C}, q \in H_{C} .
$$

We need the simple properties of $\Psi$ :

$$
\begin{gathered}
\Psi(x-t, z, q, t)=\psi(x, z, q) \psi(t, z, q)^{-1}, \\
\psi(x+1, z, q)=\psi(x, z, q) \psi(1, z, q), \\
\varphi(x+1, z, q)=\varphi_{1}(1, z, q) \vartheta(x, z, q)+\varphi_{2}(1, z, q) \varphi(x, z, q) \\
\vartheta(x+1, z, q)=\vartheta_{2}(1, z, q) \vartheta(x, z, q)+\vartheta_{1}(1, z, q) \varphi(x, z, q)
\end{gathered}
$$

The $2 \times 2$-matrix valued fundamental solution of the following equation

$$
J y^{\prime}+V y-z y=f, \quad y(0, z, q)=I, \quad z \in \mathbb{C}, q \in H_{C}
$$


has the form

$$
y(x, z)=\psi(x, z, q)-\int_{0}^{x} \Psi(x-t, z, q, t) J f(t) d t .
$$

Introduce the Banach spaces $\ell^{p}=\left\{f=\left\{f_{n}\right\}_{\mathbb{Z}}, \quad\|f\|_{(p)}^{p} \equiv \sum\left|f_{n}\right|^{p}<\infty\right\}, p \geqslant 1$, and the ball $B_{C}(q, r)=\{p:\|q-p\|<r\} \subset H_{C}, r>0$. In analogy to the notation $O(1 / n)$ we use the notation $\ell^{d}(n), d \geqslant 1$, for an arbitrary sequence of numbers, which is an element of $\ell^{d}$ (see $[\mathrm{PT}])$. For instance,

$$
a_{n}=b_{n}+\ell^{d}(n) \quad \text { is equivalent to } \quad a_{n}=b_{n}+c_{n}, \quad \sum\left|c_{n}\right|^{d}<\infty .
$$

Now we will prove the basic results about the functions $\psi$.

Lemma 2.1.i) For each $q \in H_{C}$ and $z \in \mathbb{C}$ there exists a unique solution $\psi$ of Eq. (2.5) which has the form (2.8-10) and series (2.8) converge uniformly on bounded subsets of $[0,1] \times$ $\mathbb{C} \times H_{C}$. For each $x \in[0,1]$ the function $\psi(x, z, q)$ is entire on $\mathbb{C} \times H_{C}$. Moreover, $\psi$ is analytic as a map from $\mathbb{C} \times H_{C}$ into $W_{1 C}^{2}(0,1)$ and the following estimates is fulfilled:

$$
|\psi(x, z, q)| \leqslant e^{|\operatorname{Im} z| x+\|q\|}
$$

If the sequence $q^{\nu}$ converges weakly to $q$ in $H_{C}$, as $\nu \rightarrow \infty$, then $\psi\left(x, z, q^{\nu}\right) \rightarrow \psi(x, z, q)$ uniformly on bounded subsets of $[0,1] \times \mathbb{C}$.

ii) Their derivatives with respect to $q$ have the forms

$$
\begin{gathered}
\partial_{1} \psi(x, z, q)=-\chi_{[0, x]}(t) \Psi(x-t, z, q, t) J_{2} \psi(t, z, q), \\
\partial_{2} \psi(x, z, q)=\chi_{[0, x]}(t) \Psi(x-t, z, q, t) J_{1} \psi(t, z, q) .
\end{gathered}
$$

iii) Let $q, q^{\prime} \in H_{C}$. Then

$$
\begin{gathered}
\left|\psi_{1}(x, z, q)\right| \leqslant \frac{\|q\|+\left\|q^{\prime}\right\|}{|z|} e^{|\operatorname{Im} z| x} . \\
\left|\psi(x, z, q)-\psi_{0}(x, z, q)\right| \leqslant \frac{\|q\|+\left\|q^{\prime}\right\|}{|z|} e^{|\operatorname{Im} z| x+\|q\|} .
\end{gathered}
$$

iv) Moreover, for any fixed $d>1 / 2$ the following asymptotic estimates are fulfilled

$$
\psi(x, z, q)=\sum_{0}^{3} \psi_{m}(x, z, q)+\left[\ell^{d}(n)\right]^{4}, \quad \psi_{m}(x, z, q)=\left[\ell^{d}(n)\right]^{m}, \quad m=1,2,3,
$$

as $n \rightarrow \infty$, uniformly on $[0,1] \times\{|z-\pi n| \leqslant \pi\} \times B_{C}(0, r)$ for any $r>0$.

Remark that $\left[\ell^{d}(n)\right]^{2}$ in (2.29) means that there exist two sequences $\beta=\left\{\beta_{n}(q)\right\}, \alpha=$ $\left\{\alpha_{n}(q)\right\} \in \ell^{2}$ such that $\|\beta\|_{(d)} \leqslant 1,\|\alpha\|_{(d)} \leqslant 1$, and $\psi_{2}(x, z, q)=\left[\ell^{d}(n)\right]^{2}=\alpha_{n}(q) \beta_{n}(q) O(1)$, as $n \rightarrow \infty$, uniformly on $[0,1] \times\{|z-\pi n| \leqslant \pi\} \times B_{C}(0, r)$.

Proof. i) In [PT] there is an analysis of the equation very closed to (2.5). Really repeating the corresponding proof in $[\mathrm{PT}]$, we have the statements of $\mathrm{i}$ ).

ii) We now show (2.25-26). Let $q=p+r$, where $p, r \in H_{C}$, then

$$
V(x)=P(x)+R(x), \quad P=p_{1} J_{1}+p_{2} J_{2}, \quad R=r_{1} J_{1}+r_{2} J_{2} .
$$


Then using (2.23), (2.1) we obtain

$$
\psi(x, z, q)=\psi(x, z, p)+\int_{0}^{1} \Psi(x-t, z, p, t)\left(-r_{1}(t) J_{2}+r_{2}(t) J_{1}\right) \psi(t, z, q) d t
$$

which yields (2.25-26), as $r \rightarrow 0$ since the functions $\varphi, \vartheta$ are entire.

iii) Using (2.1) and integrating by part we obtain

$$
\psi_{1}(x, z, q)=(2 z)^{-1}\left(\left.V(t) e^{z J(x-2 t)}\right|_{0} ^{x}-\int_{0}^{x} V^{\prime}(t) e^{z J(x-2 t)} d t\right)
$$

which yields

$$
\begin{aligned}
& \left|\psi_{1}(x, z, q)\right| \leqslant|2 z|^{-1}\left(\left|V(x) e^{-z J x}-V(0) e^{z J x}\right|+e^{|\operatorname{Im} z| x}\left\|q^{\prime}\right\|\right) \\
& \leqslant \frac{1}{2|z|} e^{|\operatorname{Im} z| x}\left(2 \sup |V(x)|+\left\|q^{\prime}\right\|\right) \leqslant \frac{1}{|z|} e^{|\operatorname{Im} z| x}\left(\|q\|+\left\|q^{\prime}\right\|\right) .
\end{aligned}
$$

iv) Introduce the space of continuous functions $C(0,1)$ equipped with sup-norm $\|\cdot\|_{c}$. For each function $q \in H_{C}$ and any $z \in \mathbb{C}$ define the linear operators $L(z, q)$ acting in $C(0,1)$ by the formula

$$
(L(z, q) f)(x)=\int_{0}^{x} e^{-z J(x-t)} J V(t) f(t) d t, \quad f \in C(0,1) .
$$

Let $|f(x)| \leqslant\left|f_{0}(x)\right| e^{|\operatorname{Im} z| x}$. Then the standard estimates (see [PT]) yield

$$
\left.\mid(L(z, q))^{n} f\right)(x) \mid \leqslant \frac{1}{n !}\|q\|^{n} e^{|\operatorname{Im} z| x}\left\|f_{0}\right\|_{c}, \quad n \geqslant 1, \quad x \in[0,1], z \in \mathbb{C}, q \in H_{C} .
$$

Using (2.30) we deduce that for each $z \in \mathbb{C}$ and $q \in H_{C}$ there exists the inverse operator

$$
R(z, q)=(1-L(z, q))^{-1}=1+L(z, q)+L(z, q)^{2}+L(z, q)^{3}+\ldots,
$$

and this series converge uniformly on bounded subsets of $[0,1] \times \mathbb{C} \times H_{C}$. Moreover, the following estimate is fulfilled:

$$
\|R(z, q) f\|_{c} \leqslant\left\|f_{0}\right\|_{c} e^{|\operatorname{Im} z|+\|q\|}, \quad f \in C(0,1) .
$$

Using (2.8) we have

$$
\left|\psi(x, z, q)-\psi_{0}(x, z, q)\right| \leqslant\left\|R(z, q) \psi_{1}\right\|_{c} \leqslant \frac{\|q\|+\left\|q^{\prime}\right\|_{1}}{|z|} e^{|\operatorname{Im} z|+\|q\|} .
$$

iv) Due to (2.8) we obtain

$$
\psi(x, z, q)=\sum_{0}^{3} \psi_{m}(x, z, q)+R(z, q) \psi_{4}(x, z, q)
$$


Assume that $\psi_{m}(x, z, q)=\left[\ell^{d}(n)\right]^{m}$, then using formulas (2.31) for $\psi_{m}$ we get (2.29). We have $\psi_{m}=\left(L \psi_{0}\right)^{m}$ and in [K8] the author found the asymptotics of $\psi_{m}=\left(L \psi_{0}\right)^{m}$ where $V$ is the function. The same argument is true for the matrix case.

We find $\partial M$ where $M(z, q)=\psi(1, z, q)$. Let $\vartheta=\vartheta(t, z, q), \varphi=\varphi(t, z, q)$, and $\widetilde{\vartheta}=$ $\vartheta(1, z, q), \widetilde{\varphi}=\varphi(1, z, q)$. Using $(2.18-19)$ we obtain

$$
\Psi(1, z, q, t) \equiv\left(\begin{array}{cc}
\vartheta_{1}(1, z, q, t) & \varphi_{1}(1, z, q, t) \\
\vartheta_{2}(1, z, q, t) & \varphi_{2}(1, z, q, t)
\end{array}\right)=\left(\begin{array}{cc}
\vartheta_{1} & \varphi_{1} \\
\vartheta_{2} & \varphi_{2}
\end{array}\right)\left(\begin{array}{cc}
\widetilde{\vartheta}_{1} & \widetilde{\varphi}_{1} \\
\widetilde{\vartheta}_{2} & \widetilde{\varphi}_{2}
\end{array}\right)\left(\begin{array}{cc}
\varphi_{2} & -\varphi_{1} \\
-\vartheta_{2} & \vartheta_{1}
\end{array}\right)
$$

and the multiplication yields

$$
\begin{gathered}
\Psi(1, z, q, t)= \\
\left(\begin{array}{ll}
\varphi_{2}\left(\vartheta_{1} \widetilde{\vartheta}_{1}+\varphi_{1} \widetilde{\vartheta}_{2}\right)-\vartheta_{2}\left(\vartheta_{1} \widetilde{\varphi}_{1}+\varphi_{1} \widetilde{\varphi}_{2}\right) & -\varphi_{1}\left(\vartheta_{1} \widetilde{\vartheta}_{1}+\varphi_{1} \widetilde{\vartheta}_{2}\right)+\vartheta_{1}\left(\vartheta_{1} \widetilde{\varphi}_{1}+\varphi_{1} \widetilde{\varphi}_{2}\right) \\
\varphi_{2}\left(\vartheta_{2} \widetilde{\vartheta}_{1}+\varphi_{2} \widetilde{\vartheta}_{2}\right)-\vartheta_{2}\left(\vartheta_{2} \widetilde{\varphi}_{1}+\varphi_{2} \widetilde{\varphi}_{2}\right) & -\varphi_{1}\left(\vartheta_{2} \widetilde{\vartheta}_{1}+\varphi_{2} \widetilde{\vartheta}_{2}\right)+\vartheta_{1}\left(\vartheta_{2} \widetilde{\varphi}_{1}+\varphi_{2} \widetilde{\varphi}_{2}\right)
\end{array}\right) .
\end{gathered}
$$

(2.26-27) together with (2.18) imply

$$
\begin{gathered}
\left(\partial_{1} M(z, q)\right)(t)=-M(z, q) \psi(t, z, q)^{-1} J_{2} \psi(t, z, q), \\
\left(\partial_{2} M(z, q)\right)(t)=M(z, q) \psi(t, z, q)^{-1} J_{1} \psi(t, z, q) .
\end{gathered}
$$

We find $\left(\partial_{1} M(z, q)\right)(t)$. Define the functions $(x, y)_{n} \equiv\left(J_{n} x, y\right), x, y \in \mathbb{R}^{2}, n=1,2$. We have

$$
\left(\partial_{1} M(z, q)\right)(t)=M\left(\begin{array}{cc}
\varphi_{1} & -\varphi_{2} \\
-\vartheta_{1} & \vartheta_{2}
\end{array}\right)\left(\begin{array}{cc}
\vartheta_{1} & \varphi_{1} \\
\vartheta_{2} & \varphi_{2}
\end{array}\right)=\left(\begin{array}{cc}
\widetilde{\vartheta}_{1} & \widetilde{\varphi}_{2} \\
\widetilde{\vartheta}_{2} & \widetilde{\varphi}_{2}
\end{array}\right)\left(\begin{array}{cc}
(\vartheta, \varphi)_{1} & (\varphi, \varphi)_{1} \\
-(\vartheta, \vartheta)_{1} & -(\vartheta, \varphi)_{1}
\end{array}\right)
$$

then

$$
\left(\partial_{1} M(z, q)\right)(t)=\left(\begin{array}{cc}
\widetilde{\vartheta}_{1}(\vartheta, \varphi)_{1}-\widetilde{\varphi}_{1}(\vartheta, \vartheta)_{1} & \widetilde{\vartheta}_{1}(\varphi, \varphi)_{1}-\widetilde{\varphi}_{1}(\vartheta, \varphi)_{1} \\
\widetilde{\vartheta}_{2}(\vartheta, \varphi)_{1}-\widetilde{\varphi}_{2}(\vartheta, \vartheta)_{1} & \widetilde{\vartheta}_{2}(\varphi, \varphi)_{1}-\widetilde{\varphi}_{2}(\vartheta, \varphi)_{1}
\end{array}\right)
$$

In order to find $\left(\partial_{2} M(z, q)\right)(t)$ we obtain again

$$
\left(\partial_{2} M(z, q)\right)(t)=M\left(\begin{array}{cc}
\varphi_{2} & \varphi_{1} \\
-\vartheta_{2} & -\vartheta_{1}
\end{array}\right)\left(\begin{array}{cc}
\vartheta_{1} & \varphi_{1} \\
\vartheta_{2} & \varphi_{2}
\end{array}\right)=\left(\begin{array}{cc}
\widetilde{\vartheta}_{1} & \widetilde{\varphi}_{2} \\
\widetilde{\vartheta}_{2} & \widetilde{\varphi}_{2}
\end{array}\right)\left(\begin{array}{cc}
(\vartheta, \varphi)_{2} & (\varphi, \varphi)_{2} \\
-(\vartheta, \vartheta)_{2} & -(\vartheta, \varphi)_{2}
\end{array}\right),
$$

then

$$
\left(\partial_{2} M(z, q)\right)(t)=\left(\begin{array}{cc}
\tilde{\vartheta}_{1}(\vartheta, \varphi)_{2}-\widetilde{\varphi}_{1}(\vartheta, \vartheta)_{2} & \widetilde{\vartheta}_{1}(\varphi, \varphi)_{2}-\widetilde{\varphi}_{1}(\vartheta, \varphi)_{2} \\
\widetilde{\vartheta}_{2}(\vartheta, \varphi)_{2}-\widetilde{\varphi}_{2}(\vartheta, \vartheta)_{2} & \widetilde{\vartheta}_{2}(\varphi, \varphi)_{2}-\widetilde{\varphi}_{2}(\vartheta, \varphi)_{2}
\end{array}\right) .
$$

Define the function $\Delta_{1}(z, q)=(1 / 2) \operatorname{Tr} \psi_{2}(1, z, q)$ and rewrite one in the needed form. We have $V\left(t_{1}\right) V\left(t_{2}\right)=P_{1}\left(t_{1}, t_{2}\right) I+P_{2}\left(t_{1}, t_{2}\right) J$, where

$$
P_{1}\left(t_{1}, t_{2}\right)=q_{1}\left(t_{1}\right) q_{1}\left(t_{2}\right)+q_{2}\left(t_{1}\right) q_{2}\left(t_{2}\right), \quad P_{2}\left(t_{1}, t_{2}\right)=q_{1}\left(t_{1}\right) q_{2}\left(t_{2}\right)-q_{1}\left(t_{2}\right) q_{2}\left(t_{1}\right) .
$$

Then using (2.13) we obtain

$$
\Delta_{1}(z, q)=\frac{1}{2} \operatorname{Tr} \int_{0}^{1} d t_{1} \int_{0}^{t_{1}}(I \cos \xi-J \sin \xi)\left(P_{1}\left(t_{1}, t_{2}\right) I+P_{2}\left(t_{1}, t_{2}\right) J\right) d t_{2}, \quad \xi=z\left(1-2 t_{1}+2 t_{2}\right),
$$


which yields

$$
\Delta_{1}(z, q)=\int_{0}^{1} d t_{1} \int_{0}^{t_{1}}\left(P_{1}\left(t_{1}, t_{2}\right) \cos \xi+P_{2}\left(t_{1}, t_{2}\right) \sin \xi\right) d t_{2}, \quad \xi=z\left(1-2 t_{1}+2 t_{2}\right) .
$$

Lemma 2.2.i) The functions $\Delta(z, q)$ is entire on $\mathbb{C} \times H_{C}$ and has the form

$$
\Delta(z, q)=\cos z+\Delta_{1}(z, q)+\Delta_{2}(z, q), \quad \Delta_{2}(z, q)=\frac{1}{2} \operatorname{Tr} \sum_{n \geqslant 2} \psi_{2 n}(1, z, q),
$$

where series converge uniformly on bounded subsets of $\mathbb{C} \times H_{C}$ and the estimate is fulfilled:

$$
|\Delta(z, q)| \leqslant e^{|\operatorname{Im} z| x+\|q\|}
$$

If the sequence $q^{\nu}$ converges weakly to $q$ in $H_{C}$, as $\nu \rightarrow \infty$, then $\Delta\left(z, q^{\nu}\right) \rightarrow \Delta(z, q)$ uniformly on bounded subsets of $\mathbb{C}$.

ii) Their derivatives with respect to $q$ have the forms

$$
\begin{gathered}
\left(\partial_{1} \Delta\right)(t, z, q)=-\frac{1}{2}\left(\vartheta_{2}(1, z, q, t)+\varphi_{1}(1, z, q, t)\right) \\
\left(\partial_{2} \Delta\right)(t, z, q)=\frac{1}{2}\left(\vartheta_{1}(1, z, q, t)-\varphi_{2}(1, z, q, t)\right) .
\end{gathered}
$$

In particular, if $\left|\gamma_{n}\right|=0$, for some $q \in H$, then $\partial \Delta(z, q)=0$ at $z=m_{n}(q)$.

iii) For fixed $z \in \mathbb{C}$ the function $\Delta(z, q)$ is even with respect to $q \in H_{C}$ and

$$
\Delta(z,-q)=\Delta(z, q), \quad q \in H_{C}
$$

Proof. i) Introduce the set of matrises $\mathcal{A}=\left\{a: a=p J_{1}+q J_{2}, p, q \in \mathbb{C}\right\}$. Below we need simple properties of $\mathcal{A}$. Let $a, a_{1}, a_{2} \in \mathcal{A}$ then we have $\operatorname{Tr} a=0$ and

$$
a a_{1} a_{2} \in \mathcal{A}, \quad J a \in \mathcal{A} .
$$

Relations (2.44) yield $\operatorname{Tr} V\left(t_{1}\right) \ldots V\left(t_{2 n+1}\right)$ for any $t_{1}, \ldots t_{2 n+1} \in[0,1]$ and then

$$
\operatorname{Tr} e^{-z J\left(x-t_{1}+2 t_{2} \ldots-2 t_{2 n+1}\right)} J V\left(t_{1}\right) \ldots V\left(t_{2 n+1}\right)=0,
$$

for any $x, t_{1}, \ldots t_{2 n+1} \in[0,1]$, which together with (2.16) implies

$$
\operatorname{Tr} \psi_{2 n+1}(x, z, q)=0,
$$

for any $(x, z, q) \in[0,1] \times \mathbb{C} \times H_{C}$. Series (2.8) converge uniformly and absolutely, then (2.45) implies (2.39). Moreover, using the properties of the fundamental solutions (Lemma 2.1) we obtain the results for the Lyapunov function $\Delta$ stated in i).

ii) Combining (2.36-37) and (2.33) we have (2.41-42). It is well known that in each closed gap $\bar{\gamma}_{n}$ there exist both the zero of $\vartheta(1, z, q)$ and $\varphi(1, z, q)$. Then if $\left|g_{n}\right|=0$ then by (2.41-42), $\partial \Delta(z, q)=0$ at $z=m_{n}(q)$. 
iii) Formula (2.39) yields identity (2.43).

Introduce the Fourier transformation $\Phi: H_{C} \rightarrow \ell_{C}^{2} \oplus \ell_{C}^{2}$ by the formulas:

$$
\Phi f=\left\{\hat{f}_{n}\right\}, \quad \hat{f}_{n}=\left(\begin{array}{c}
f_{n 1} \\
f_{n 2}
\end{array}\right)=\int_{0}^{1} \psi_{0}(x, 2 \pi n)^{*} f(x) d x=\left(\begin{array}{c}
f_{1(n c)}+f_{2(n s)} \\
-f_{1(n s)}+f_{2(n c)}
\end{array}\right),
$$

$f_{m(n c)}=\int_{0}^{1} f_{m}(x) \cos 2 \pi n x d x, \quad f_{m(n s)}=\int_{0}^{1} f_{m}(x) \sin 2 \pi n x d x, \quad f(x)=\sum \psi_{0}(x, 2 \pi n) \hat{f}_{n}$, $\mathrm{m}=1,2$, and let $\hat{q}_{n}^{2} \equiv\left(q_{1(n c)}+q_{2(n s)}\right)^{2}+\left(-q_{1(n s)}+q_{2(n c)}\right)^{2}$. We find now the asymptotics estimates of the Lyapunov function.

Lemma 2.3. Let $q \in H_{C}$. Then following identity and the asymptotic estimates, for any fixed $d>1$, are fulfilled:

$$
\Delta_{1}(\pi n, q)=\frac{(-1)^{n}}{2} \hat{q}_{n}^{2},
$$

and

$$
\begin{gathered}
\Delta_{1}(z, q)=\ell^{d}(n), \quad \Delta_{2}(z, q)=\left(\ell^{d}(n)\right)^{2}, \\
\left(\partial_{1} \Delta(z, q)\right)(t)=\int_{0}^{1}\left(q_{1}(x+t) \cos z(2 x-1)+q_{2}(x+t) \sin z(2 x-1)\right) d x+\ell^{d}(n), \\
\left(\partial_{2} \Delta(z, q)\right)(t)=\int_{0}^{1}\left(q_{1}(x+t) \sin z(2 x-1)-q_{2}(x+t) \cos z(2 x-1)\right) d x+\ell^{d}(n), \\
\left(\frac{\partial^{m}}{\partial z^{m}} \partial \Delta(z, q)\right)(t)=\ell^{2}(n), \quad \text { at } \quad z=\pi n, m \geqslant 1, \\
(\partial \Delta(z, q))(t)=d_{q} \hat{q}_{n}^{2}+\ell^{d}(n), \quad \text { if } z-\pi n=\ell^{2}(n),
\end{gathered}
$$

as $|z| \rightarrow \infty,|z-\pi n| \leqslant \pi$. These asymptotics are infinitely differentiable with respect to $z$ and are satisfied uniformly on bounded subsets of $[0,1] \times H_{C}$.

Proof. We have $\Delta_{1}(\pi n, q)=(-1)^{n} \Delta_{1 c}+(-1)^{n} \Delta_{1 s}$, where

$$
\begin{aligned}
& \Delta_{1 c}=\int_{0}^{1} d t_{1} \int_{0}^{t_{1}}\left(q_{1}\left(t_{1}\right) q_{1}\left(t_{2}\right)+q_{2}\left(t_{1}\right) q_{2}\left(t_{2}\right)\right) \cos 2 \pi n\left(t_{2}-t_{1}\right) d t_{2}, \\
& \left.\Delta_{1 s}=\int_{0}^{1} d t_{1} \int_{0}^{t_{1}}\left(q_{1}\left(t_{1}\right) q_{2}\left(t_{2}\right)-q_{1}\left(t_{2}\right) q_{2}\left(t_{1}\right)\right) \sin 2 \pi n\left(t_{2}-t_{1}\right)\right) d t_{2} .
\end{aligned}
$$

Then the simple integration implies

$$
\Delta_{1 c}=\frac{1}{2}\left(q_{1(n c)}^{2}+q_{1(n s)}^{2}+q_{2(n c)}^{2}+q_{2(n s)}^{2}\right)
$$

and

$$
\left.\left.\Delta_{1 s}=\int_{0}^{1} d t_{1} \int_{0}^{t_{1}} q_{1}\left(t_{1}\right) q_{2}\left(t_{2}\right) \sin 2 \pi n\left(t_{2}-t_{1}\right)\right) d t_{2}-\int_{0}^{1} d t_{1} \int_{0}^{t_{1}} q_{1}\left(t_{2}\right) q_{2}\left(t_{1}\right) \sin 2 \pi n\left(t_{2}-t_{1}\right)\right) d t_{2}
$$




$$
\left.\left.=\int_{0}^{1} d t_{1} \int_{0}^{t_{1}} q_{1}\left(t_{1}\right) q_{2}\left(t_{2}\right) \sin 2 \pi n\left(t_{2}-t_{1}\right)\right) d t_{2}-\int_{0}^{1} d t_{2} \int_{t_{2}}^{1} q_{1}\left(t_{2}\right) q_{2}\left(t_{1}\right) \sin 2 \pi n\left(t_{2}-t_{1}\right)\right) d t_{1} .
$$

Then

$$
\left.\Delta_{1 s}=\int_{0}^{1} d t_{1} \int_{0}^{1} q_{1}\left(t_{1}\right) q_{2}\left(t_{2}\right) \sin 2 \pi n\left(t_{2}-t_{1}\right)\right) d t_{2}=q_{1(n c)} q_{2(n s)}-q_{1(n s)} q_{2(n c)},
$$

and summing (2.52-53) we get (2.46).

Lemma 2.1 gives $(2.47)$.

Substituting (2.29), (2.12) into (2.34-35), we obtain (2.48-49).

Using (2.47-48) we get (2.50-51).

\section{Functions}

For $q$ we define the new function $\widetilde{q}$ and the constant $N_{q}$ by the following formulas

$$
\widetilde{q}(x)=\sum_{-M}^{M} \psi_{0}(x, 2 \pi n) \hat{q}_{n}, \quad N_{q}=24\left(\|q\|+\left\|\widetilde{q}^{\prime}\right\|\right) e^{2\|q\|}
$$

where $M$ is defined by the following condition: $\|q-\widetilde{q}\| \leqslant \varepsilon$. Define the contur $c_{n}(r)=\{z$ : $|z-\pi n|=r\}, r>0$.

Lemma 3.1. i) Let $q \in H$ and $\varepsilon_{q}=4^{-4} \exp (-3\|q\|)$. Then for each integer $N>N_{q}$ and any $p \in B_{C}\left(q, \varepsilon_{q}\right)$ the function $\varphi_{1}(1, z, p)$ has exactly $2 N+1$ roots, counted with multiplicities, in the disc $\{z:|z|<\pi(N+(1 / 2))\}$ and for each $|n|>N$, exactly one simple root in the domain $\{z:|z-\pi n|<1\}$. There are no other roots.

ii) Each function $m_{n}(\cdot), n \in \mathbb{Z}$, is compact and real analytic on $H$. Its gradient is given by the formula

$$
\left(d_{q} m_{n}(q)\right)(x)=-\frac{\left((\varphi(t, z, q), \varphi(t, z, q))_{1},(\varphi(t, z, q), \varphi(t, z, q))_{2}\right)}{\dot{\varphi}_{1}(1, z, q) \varphi_{2}(1, z, q)}, \quad z=m_{n}(q),
$$

and

$$
\|\varphi(\cdot, z, q)\|^{2}=\dot{\varphi}_{1}(1, z, q) \varphi_{2}(1, z, q)>0, \quad z=m_{n}(q) .
$$

Moreover, for any fixed $d>1$ the following asymptotic estimates are fulfilled:

$$
\begin{gathered}
m_{n}(q)=\pi n-q_{1(c n)}-q_{2(s n)}+\ell^{d}(n), \\
\left(d_{q} m_{n}(q)\right)(x)=-(\cos 2 \pi n x, \sin 2 \pi n x)+\ell^{2}(n),
\end{gathered}
$$

as $n \rightarrow \infty$, uniformly on $[0,1] \times B_{C}\left(p, \varepsilon_{p}\right)$ for each fixed $p \in H$.

Proof. i) Let $N_{1}>N$ be another integer and let $z$ belong to the contours $|z|=\pi(N+$ $(1 / 2)),|z|=\pi\left(N_{1}+(1 / 2)\right), c_{n}(1),|n|>N$. Recall that for $q$ we defined the functions $\widetilde{q}$ and the integer $M \geqslant 1$ (see (3.1)) such that $\|q-\widetilde{q}\| \leqslant \varepsilon=\varepsilon_{q}$. Using the estimates (2.28) we get

$$
\left|\varphi_{1}(1, z, \widetilde{q})+\sin z\right| \leqslant \frac{\|q\|+\left\|\widetilde{q}^{\prime}\right\|}{|z|} \exp \{\|q\|+|\operatorname{Im} z|\} .
$$


We have the equality

$$
\varphi_{1}(1, z, p)-\varphi_{1}(1, z, \widetilde{q})=\int_{0}^{1}\left(\partial \varphi_{1}(1, z, \widetilde{q}+t v), v\right) d t, \quad v=p-\widetilde{q}
$$

where $(\cdot, \cdot)$ is the scalar product in $H_{C}$. Identities $(2.25-26)$ together with the estimates (2.24) yield

$$
\left|\partial \varphi_{1}(1, z, \widetilde{q}+t v)\right| \leqslant e^{|\operatorname{Im} z|+2|| \widetilde{q}+t v||} \leqslant e^{|\operatorname{Im} z|+2|| q||+4 \varepsilon}, \quad t \in(0,1),
$$

since $\|v\| \leqslant\|p-q\|+\|q-\widetilde{q}\| \leqslant 2 \varepsilon$. The substitution of this estimate into (3.7) implies

$$
\left|\varphi_{1}(1, z, p)-\varphi_{1}(1, z, \widetilde{q})\right| \leqslant 2 \varepsilon e^{|\operatorname{Im} z|+2|| q||+4 \varepsilon} .
$$

Using (3.6),(3.9), and the simple estimate $\exp |\operatorname{Im} z|<4|\sin z|$ on all contours (see [PT]) we obtain

$$
\begin{gathered}
\left|\varphi_{1}(1, z, p)+\sin z\right| \leqslant\left|\varphi_{1}(1, z, p)-\varphi_{1}(1, z, \widetilde{q})\right|+\left|\varphi_{1}(1, z, \widetilde{q})+\sin z\right| \\
\leqslant e^{|\operatorname{Im} z|}\left(2 \varepsilon e^{2|| q \mid+4 \varepsilon}+\frac{1}{36}\right)<\frac{1}{2}|\sin z| .
\end{gathered}
$$

Hence, by Rouché's theorem, $\varphi_{1}(1, z, r)$ has as many roots, counted with multiplicities, as $\sin z$ in each of the bounded domains and the remaining unbounded region. Since $\sin z$ has only simple roots at $\pi n, n \in \mathbb{Z}$, and since $N_{1}>N$ can be chosen arbitrarily large, the point i) of Lemma 3.1 follows.

ii) First we prove (3.3) which shows that the zero $m_{n}(q)$ is simple for $q \in H$. In fact, we repeat the case of the potential from [PT]. The differentiation of Eq. (1.3) with respect to $z$ implies

$$
J \dot{\varphi}^{\prime}+V \dot{\varphi}=\varphi+z \dot{\varphi} .
$$

Multiplying this equation by $\varphi$, the original equation by $\dot{\varphi}$ and taking the difference we deduce that

$$
\varphi^{2}=\left(J \dot{\varphi}^{\prime}, \varphi\right)-(J \varphi, \dot{\varphi})=W(\dot{\varphi}, \varphi)^{\prime} .
$$

Let $m_{n}=m_{n}(q), \varphi\left(x, m_{n}\right)=\varphi\left(x, m_{n}, q\right)$. Then

$$
\begin{aligned}
& \int_{0}^{1} \varphi\left(x, m_{n}\right)^{2} d x=\int_{0}^{1} W\left(\dot{\varphi}\left(x, m_{n}\right), \varphi\left(x, m_{n}\right)\right)^{\prime} d x \\
= & \left.\left.W\left(\dot{\varphi}\left(x, m_{n}\right), \varphi\left(x, m_{n}\right)\right)\right|_{0} ^{1}=\dot{\varphi}_{1}\left(1, m_{n}\right) \varphi_{2}\left(1, m_{n}\right)\right),
\end{aligned}
$$

since $\varphi_{1}(0, z)$ and $\dot{\varphi}(0, z)$ vanish for all $z$ and $\varphi_{1}(1, z)$ vanishes for a Dirichlet eigenvalue $z=m_{n}$. The integral is equal to $\|\varphi(\cdot, z)\|^{2}$ since $\varphi$ is real for real $z$.

In order to prove compactness of $m_{n}(q)$ suppose that the sequence $q^{\nu}, \nu \geqslant 1$, converges weakly to $q$ in $H$. For small $\delta>0$ we introduce the intervals

$$
I_{n}=\left[m_{n}(q)-\delta, m_{n}(q)+\delta\right] \subset \mathbb{R}, \quad-N \leqslant n \leqslant N
$$


If $\delta$ is sufficiently small, then these intervals are all disjoint. The function $\varphi_{1}(1, z, q)$ changes sign on each of them and $\left|\dot{\varphi}_{1}(1, z, q)\right|>2 B, \quad z \in \cup I_{n}$, for some $B>0$ since $m_{n}(q)$ is a simple root.

As $\nu \rightarrow \infty$, the functions $\varphi_{1}\left(1, z, q^{\nu}\right) \rightarrow \varphi_{1}(1, z, q)$ and $\dot{\varphi}_{1}\left(1, z, q^{\nu}\right) \rightarrow \dot{\varphi}_{1}(1, z, q)$ converge uniformly on $\cup I_{n}$ by Lemma 2.1. Hence, for sufficiently large $\nu$, the functions $\varphi_{1}\left(1, z, q^{\nu}\right)$ also change sign and $\left|\dot{\varphi}_{1}\left(1, z, q^{\nu}\right)\right|>B$ on $\cup I_{n}$, so they must all have one root in each of these intervals, for $\nu$ sufficiently large. Hence $\varphi_{1}\left(1, z, q^{\nu}\right)$ has exactly one root $m_{n}\left(q^{\nu}\right)$ in each segment $I_{n},|n| \leqslant N$. This yields $\left|m_{n}\left(q^{\nu}\right)-m_{n}(q)\right|<\delta,|n| \leqslant N$, for all sufficiently large $\nu$. It follows that $m_{n}\left(q^{\nu}\right) \rightarrow m_{n}(q)$, as $\nu \rightarrow \infty$, since $N$ and $\delta>0$ were arbitrary. Thus, $m_{n}(q)$ is a compact functions of $q$.

To prove real analyticity, we fix $p \in H$. Then $\dot{\varphi}_{1}\left(1, z_{n}(p), p\right) \neq 0$. Now, the implicit function theorem guarantees the existence of a unique continuous function $\widetilde{m}_{n}$ defined on some small neighborhood $W \subset H$ of $p$ and such that

$$
\varphi_{1}\left(1, \widetilde{m}_{n}(q), q\right)=0, \quad \widetilde{m}_{n}(p)=m_{n}(p)
$$

on $W$. Furthermore, $\widetilde{m}_{n}(q)$ is real analytic. On the other hand, $m_{n}(q)$ is also a continuous function on $W$ satisfying $\varphi_{1}\left(1, m_{n}(q), q\right)=0$. Therefore, by uniqueness, $\widetilde{m}_{n}(q)=m_{n}(q)$ on $W$, and so $m_{n}(q)$ is real analytic.

To calculate the gradient, we observe that $\varphi_{1}\left(1, m_{n}(q), q\right)=0$. Hence

$$
0=d_{q}\left\{\varphi_{1}\left(1, m_{n}(q), q\right)\right\}=\dot{\varphi}_{1}\left(1, m_{n}(q), q\right) d_{q} m_{n}+\partial \varphi_{1}(1, z, q), \quad z=m_{n}(q) .
$$

By (2.36-37), the second term has the form

$$
\left(\partial_{j} \varphi_{1}(1, z, q)\right)(x)=\vartheta_{1}(1, z, q)(\varphi(t, z, q), \varphi(t, z, q))_{j}, \quad z=m_{n}(q), \quad j=1,2,
$$

and the last two identities give

$$
\left(d_{q} m_{n}\right)(x)=-\frac{\vartheta_{1}\left(1, m_{n}, q\right)}{\dot{\varphi}_{1}\left(1, m_{n}(q), q\right)}\left((\varphi(t, z, q), \varphi(t, z, q))_{1},(\varphi(t, z, q), \varphi(t, z, q))_{2}\right),
$$

which implies (3.2).

Asymptotics of the quasimomentum $k(z)$ (see Sect.1) yields $m_{n}=\pi n+o(1), n \rightarrow \pm \infty$ for $p \in H$. Moreover, by i), $\left|m_{n}(p)-\pi n\right|<1$ for $|n|>N, p \in B_{C}\left(q, \varepsilon_{q}\right)$. We improve last results. Relation (2.29) yields

$$
\varphi_{1}(1, z, q)=-\sin z-\int_{0}^{1}\left(q_{1}(x) \cos z(2 x-1)+q_{2}(x) \sin z(2 x-1)\right) d x+\ell^{d}(n), \quad|z-\pi n| \leqslant 1,
$$

as $n \rightarrow \infty$. Hence

$$
0=\varphi_{1}\left(1, m_{n}, q\right)=-\sin m_{n}-\int_{0}^{1}\left(q_{1}(x) \cos m_{n}(2 x-1)+q_{2}(x) \sin m_{n}(2 x-1)\right) d x+\ell^{d}(n),
$$

which implies (3.4). Using (3.2) and (2.29) we obtain

$$
d_{q} m_{n}=-\left(1+\ell^{2}(n)\right)\left(\sin ^{2} \pi n t-\cos ^{2} \pi n t+\ell^{2}(n),-2 \sin \pi n t \cos \pi n t+\ell^{2}(n)\right)
$$


which implies (3.5).

For $q \in H$ we introduce the domain $U_{n}^{ \pm}(q)=\left\{z:\left|z-z_{n}^{0}(q)\right|<r_{n}^{ \pm}\right\}$, and the contours

$\beta_{n}(q)=\left\{z:\left|z-z_{n}^{0}(q)\right|=\left(r_{n}^{-}+r_{n}^{+}\right) / 2\right\}, \quad r_{n}^{ \pm}=\frac{1}{2}\left|\gamma_{n}(q)\right|+\frac{2 \pm 1}{6} \min \left\{\left|\sigma_{n}\right|,\left|\sigma_{n+1}\right|\right\}, \quad n \in \mathbb{Z}$.

In the following Lemma we study general properties of $z_{n}^{ \pm}(q)$.

Lemma 3.2. i) Let Let $q \in H$ and $\varepsilon_{q}=4^{-4} \exp (-3\|q\|)$. Then for each integer $N>N_{q}$ and any $p \in B_{C}\left(q, \varepsilon_{q}\right)$ the function $\Delta(z, p)^{2}-1$ has exactly $4 N+2$ roots, counted with multiplicities, in the disc $\{z:|z|<\pi(N+(1 / 2))\}$ and for each $|n|>N$, exactly 2 roots $\widetilde{z}_{n}^{ \pm}(q)$ in the domain $\{z:|z-\pi n|<1\}$. There are no other roots, and uniformly on $B_{C}\left(q, \varepsilon_{q}\right)$ the following asymptotics are fulfilled:

$$
\widetilde{z}_{n}^{ \pm}(p)=\pi n \pm \sqrt{\hat{p}_{n}^{2}}+\ell^{d}(n), \quad|n| \rightarrow \infty .
$$

ii) For each $p \in H$ and any $n \in \mathbb{Z}$ there exists $\varepsilon \in(0,1]$ such that for any $q \in B_{C}(p, \varepsilon)$ the function $\Delta(z, q)^{2}-1$ has exactly 2 roots, counted with multiplicities, in the disc $U_{n}^{ \pm}(p)$.

iii) Each function $z_{n}^{ \pm}(\cdot), n \in \mathbb{Z}$, is compact on $H$.

Remark. Note that the function $\widetilde{z}_{n}^{ \pm}(q)$ is complicated and in not analytic. Below we need only their sum $\widetilde{z}_{n}^{-}(q)+\widetilde{z}_{n}^{+}(q)=z_{n}^{-}(q)+z_{n}^{+}(q)$ which is analytic, and we study this function in Lemma 3.3.

Proof. i) Fix $N>N_{q}$, and let $N_{1}>N$ be another integer. Consider the contours

$$
|z|=\pi(N+(1 / 2)), \quad|z|=\pi\left(N_{1}+(1 / 2)\right), \quad|z-\pi n|=1, \quad|n|>N .
$$

Using (2.28) we have the following estimates for the function $\widetilde{q}, \varepsilon=\varepsilon_{q}$ (see (3.1))

$$
|\Delta(z, \widetilde{q})-\cos z| \leqslant \frac{\|q\|+\left\|\widetilde{q}^{\|}\right\|}{|z|} e^{\|q\|+|\operatorname{Im} z|}<\frac{e^{|\operatorname{Im} z|-\|q\|}}{24 \pi} .
$$

We have the equality

$$
\Delta(z, p)-\Delta(z, \widetilde{q})=\int_{0}^{1}(\partial \Delta(z, \widetilde{q}+t v), v) d t, \quad v=p-\widetilde{q}
$$

where $(\cdot, \cdot)$ is the scalar product in $H_{C}$. Identities (2.41-42) and estimates $(2.24)$ yield

$$
|\partial \Delta(z, \widetilde{q}+t v)| \leqslant e^{|\operatorname{Im} z|+2|| \widetilde{q}+t v \|}
$$

and the simple inequalities $\|v\| \leqslant\|p-q\|+\|q-\widetilde{q}\| \leqslant 2 \varepsilon$ and $\|\widetilde{q}+t v\| \leqslant\|q\|+2 \varepsilon$ give

$$
|\Delta(z, p)-\Delta(z, \widetilde{q})| \leqslant 2 \varepsilon e^{|\operatorname{Im} z|+2|| q||+4 \varepsilon} .
$$

Using estimates (2.40) we obtain

$$
\left.\left|\left(1-\Delta^{2}(z, p)\right)-\sin ^{2} z\right| \leqslant\left|\Delta^{2}(z, p)-\Delta^{2}(z, \widetilde{q})\right|+\mid \Delta^{2}(z, \widetilde{q})\right)-\cos ^{2} z \mid
$$




$$
\leqslant 2 e^{|\operatorname{Im} z|+|| q||+\varepsilon}|\Delta(z, p)-\Delta(z, \widetilde{q})|+2 e^{|\operatorname{Im} z|+|| q \|}|\Delta(z, \widetilde{q})-\cos z| .
$$

Substituting (3.12), (3.15) and the simple estimate $\exp (|\operatorname{Im} z|) \leqslant 4|\sin z|$ as $|z-\pi m| \geqslant$ $\pi / 4, m \in \mathbb{Z}$, (see $[\mathrm{PT}]$ ) into last estimate we get

$$
\left|\left(1-\Delta^{2}(z, p)\right)-\sin ^{2} z\right| \leqslant\left(\frac{1}{32}+\frac{1}{12 \pi}\right) e^{2|\operatorname{Im} z|}<\frac{17}{18}\left|\sin ^{2} z\right|
$$

on all contours. Hence, by Rouche's theorem, $\Delta(z, q)^{2}-1$ has as many roots, counted with multiplicities, as $\sin ^{2} z$ in each of the bounded regions and the remaining unbounded domain. Since $\sin ^{2} z$ has only the roots $\pi n, n \in \mathbb{Z}$, and since $N_{1}>N$ can be chosen arbitrarily large, i) of the lemma follows, without (3.11).

Asymptotics of the quasimomentum $k(z)$ (see Sect.1) yields $z_{n}^{ \pm}(p)=\pi n+o(1), n \rightarrow \pm \infty$ for $p \in H$. Moreover, we have $\left|z_{n}^{ \pm}(p)-\pi n\right|<1$ for $|n|>N, p \in B_{C}\left(q, \varepsilon_{q}\right)$. Now we improve the last result. Using (2.47) we obtain $\Delta(z, p)=\cos z+o(1), \quad|z| \rightarrow \infty$, which implies $\widetilde{z}_{n}^{ \pm}(p)=\pi n+o(1), \quad|n| \rightarrow \infty$. We improve the last result again. Let $r_{n}=\widetilde{z}_{n}^{ \pm}-\pi n$. Then using (2.47) we deduce that

$$
(-1)^{n}=(-1)^{n} \cos r_{n}+\ell^{d}(n), \quad d>1,
$$

and $2 \sin ^{2}\left(r_{n} / 2\right)=\ell^{d}(n)$, which yields

$$
r_{n}=\ell^{d / 2}(n), \quad|n| \rightarrow \infty .
$$

Using again (2.46-47), (3.17) we get the following asymptotics estimates

$$
\begin{gathered}
2 \sin ^{2}\left(r_{n} / 2\right)=(-1)^{n} \Delta_{1}\left(\pi n+r_{n}, p\right)+\left(\ell^{d}(n)\right)^{2}, \\
(-1)^{n} \Delta_{1}\left(\pi n+r_{n}, p\right)=(1 / 2) \hat{p}_{n}^{2}+\Delta_{1}^{\prime}(\pi n, p) r_{n}+\left(\ell^{d}(n)\right)^{2},
\end{gathered}
$$

and then the last relations and (2.47) yield

$$
r_{n}^{2}=\hat{p}_{n}^{2}+2 x_{n} r_{n}+\left(\ell^{d}(n)\right)^{2}, \quad n \rightarrow \infty,
$$

where $x_{n}=(-1)^{n} \Delta_{1}^{\prime}(\pi n, p)$, which implies

$$
r_{n}=x_{n} \pm \sqrt{\hat{p}_{n}^{2}+\left(\ell^{d}(n)\right)^{2}}= \pm \sqrt{\hat{p}_{n}^{2}}+\ell^{d}(n), \quad n \rightarrow \infty
$$

Using last estimates we have (3.11).

ii) The analyticity (see Lemma 2.2) of $\Delta$ yields

$$
\sup _{z \in U_{n}^{ \pm}(p),\|q-p\|_{H_{C}} \leqslant \varepsilon}|\Delta(z, q)-\Delta(z, p)|<C_{0} \varepsilon,
$$

for some small $\varepsilon>0$ and constant $C_{0}=C_{0}(p, n)$. Introduce the constants

$$
C_{1}=\max _{z \in \partial U_{n}^{ \pm}(p)}|\Delta(z, p)|, \quad C_{2}=\min _{z \in \partial U_{n}^{ \pm}(p)}\left|\Delta(z, p)^{2}-1\right|>0 .
$$


Let $S=\varepsilon\left(C_{0} / C_{2}\right)\left(C_{0} \varepsilon+2 C_{1}\right)$, then estimate (3.21) implies

$$
\left|\left(\Delta(z, q)^{2}-1\right)-\left(\Delta(z, p)^{2}-1\right)\right|<C_{0} \varepsilon\left(C_{0} \varepsilon+2 C_{1}\right) \leqslant S\left|\Delta(\lambda, p)^{2}-1\right|,
$$

where $\|q-p\|_{C} \leqslant \varepsilon, z \in \partial U_{n}^{ \pm}(p)$, and if we take $\varepsilon$ such that $S<1$ hence by Rouche's theorem, $\Delta(z, q)^{2}-1$ has as many roots, counted with multiplicities, as $\Delta(z, p)^{2}-1$ in the disc $U_{n}^{ \pm}(p)$. Since $\Delta(z, p)^{2}-1$ has two roots, counted with multiplicities, we have proved ii).

iii) In order to verify compactness of $z_{n}^{ \pm}$suppose a sequence $q^{\nu}, \nu \geqslant 1$, converges weakly to $q$ in $H$. For some $q$ and $N>1, \varepsilon>0$, we introduce the set $I=\left(\cup I_{n}^{0}\right) \cup\left(\cup I_{n}^{ \pm}\right)$and the intervals:

$$
\begin{gathered}
I_{n}^{ \pm}=\left[z_{n}^{ \pm}(q)-\varepsilon, z_{n}^{ \pm}(q)+\varepsilon\right], \quad \text { if } \quad z_{n}^{-}(q) \neq z_{n}^{+}(q) \\
I_{n}^{0}=\left[z_{n}^{+}(q)-\varepsilon, z_{n}^{+}(q)+\varepsilon\right], \quad \text { if } \quad z_{n}^{-}(q)=z_{n}^{+}(q), \quad-N \leqslant n \leqslant N .
\end{gathered}
$$

If $\varepsilon$ is sufficiently small, then these intervals are all disjoint and contained in the disk $D=$ $\{z:|z|<r\}$ for some $r>0$. The function $f(z, q) \equiv \Delta^{2}(z, q)-1$ changes sign on each $I_{n}^{ \pm}$, that is $f(z, q)$ has a simple root on each $I_{n}^{ \pm}$and $|\dot{f}(z, q)|>B>0$ on each $I_{n}^{ \pm}$, for some $B>0$. Moreover, the function $f(z, q)$ has a root of multiplicity 2 and $|\ddot{f}(z, q)|>B>0$ on each $I_{n}^{0}$.

The entire functions $f\left(z, q^{\nu}\right)$ converge to $f(z, q)$, as $\nu \rightarrow \infty$, uniformly on $D$ by Lemma 2.2. Then, for sufficiently large $\nu$, any function $f\left(z, q^{\nu}\right)$ has a simple root in each interval $I_{n}^{ \pm}$and $f\left(z, q^{\nu}\right)$ has exactly two roots in each interval $I_{n}^{0}$, which must be the eigenvalues $z_{n}^{ \pm}\left(q^{\nu}\right)$. This yields $\left|z_{n}^{ \pm}\left(q^{\nu}\right)-z_{n}^{ \pm}(q)\right|<\varepsilon,|n| \leqslant N$, for all sufficiently large $\nu$. It follows that $z_{n}^{ \pm}\left(q^{\nu}\right) \rightarrow z_{n}^{ \pm}(q), \nu \rightarrow \infty$, since $N$ and $\varepsilon>0$ were arbitrary. Thus, $z_{n}^{ \pm}(\cdot)$ are the compact functions of $q$.

Introduce the functions

$$
F(z, q) \equiv \frac{\Delta(z, q) \Delta_{z}(z, q)}{\Delta^{2}(z, q)-1}, \quad G(z, q) \equiv \frac{\Delta(z, q) \partial \Delta(z, q)}{\Delta^{2}(z, q)-1}
$$

Recall $z_{n}^{0}=\left(z_{n}^{-}+z_{n}^{+}\right) / 2$. In order to study $g_{n 1}$ we have to consider the function $z_{n}^{0}(q), q \in H$. Lemma 3.3. Each function $z_{n}^{0}(\cdot), n \in \mathbb{Z}$, is compact and real analytic on $H$. Its gradient is given by the formulas

$$
\begin{gathered}
d_{q} z_{n}^{0}=-\frac{\partial \Delta\left(z_{n}^{-}, q\right)}{2 \Delta_{z}\left(z_{n}^{-}, q\right)}-\frac{\partial \Delta\left(z_{n}^{+}, q\right)}{2 \Delta_{z}\left(z_{n}^{+}, q\right)}, \quad z_{n}^{-} \neq z_{n}^{+}, \\
d_{q} z_{n}^{0}=-\frac{\partial \Delta_{z}\left(z_{n}^{0}, q\right)}{\Delta_{z z}\left(z_{n}^{0}, q\right)}, \quad z_{n}^{0}=z_{n}^{-}=z_{n}^{+} .
\end{gathered}
$$

Moreover,

$$
\begin{gathered}
z_{n}^{0}(q)=\pi n+\ell^{d}(n), \\
d_{q} z_{n}^{0}(q)=\ell^{2}(n),
\end{gathered}
$$

$n \rightarrow \infty$ uniformly on bounded subsets of $[0,1] \times B_{C}\left(p, \varepsilon_{p}\right)$ for any fixed $p \in H$. 
Proof. To prove real analyticity, fix $p \in H$ and consider $z_{n}^{0}(q)$ for some $n \in \mathbb{Z}$. By Lemma 2.3 , there exists $\varepsilon \in(0,1)$ such that for all $q \in B_{C}\left(p, \varepsilon_{p}\right)$ the function $\Delta(z, q)^{2}-1$ has exactly two roots $\widetilde{z}_{n}^{ \pm}(q)$ in each disc $U_{n}^{-}(p) \subset U_{n}^{+}(p)$. We have the identity

$$
z_{n}^{0}(q)=\frac{1}{2 \pi i} \int_{\beta_{n}(p)} z F(z, q) d z
$$

for all $q \in B\left(p, \varepsilon_{p}\right)$. The function $F(z, q), z \in \beta_{n}(p), q \in B\left(p, \varepsilon_{p}\right)$ has analytic extension on the ball $B_{C}\left(p, \varepsilon_{p}\right)$, then by $(3.26), z_{n}^{0}(\cdot)$ also has analytic extension on the ball $B_{C}\left(p, \varepsilon_{p}\right)$. Hence $z_{n}^{0}(\cdot)$ is a real analytic function on $H$.

In order to calculate the gradient we use the theorem of the residue. Using (3.26) and integrating by parts, we deduce that

$$
d_{q} z_{n}^{0}=\frac{1}{2 \pi i} \int_{\beta_{n}} z \partial F(z, q) d z=-\frac{1}{2 \pi i} \int_{\beta_{n}} G(z, q) d z
$$

which implies (3.22-23). Note that if $z_{n}^{-}(q)=z_{n}^{+}(q)$, then Lemma 2.2 yields $\partial \Delta\left(z_{n}^{-}, q\right)=0$.

We show (3.24-25). First, (3.11) yields (3.24). Let $q \in B_{C}\left(p, \varepsilon_{p}\right)$. Then by i) of Lemma $3.2,\left|\widetilde{z}_{n}^{ \pm}(q)-\pi n\right|<1$ for all $|n|>N_{p}$ that is the distance between $\widetilde{z}_{n}^{ \pm}(q)$ and the contour $c_{n}(r), r=3 / 2$, is greater than $1 / 2$. Rewriting integral (3.27) in the form

$$
d_{q} z_{n}^{0}=\frac{1}{2 \pi i} \int_{c_{n}(r)} z \partial F(z, q) d z=-\frac{1}{2 \pi i} \int_{c_{n}} G(z, q) d z
$$

and substituting estimates (2.5), (2.10), we obtain

$$
d_{q} z_{n}^{0}=-\frac{1}{2 \pi i} \int_{c_{n}(r)} \frac{\left(\cos z+\ell^{d}(n)\right) \partial \Delta(z, q) d z}{-\sin ^{2} z+\ell^{d}(n)}=\partial \dot{\Delta}(\pi n, q)+\ell^{d}(n)
$$

as $n \rightarrow \infty$, uniformly on bounded subsets of $[0,1] \times B_{C}\left(p, \varepsilon_{p}\right)$.

We formulate the result about $g_{n 1}$

Lemma 3.4. Each function $g_{n 1}(\cdot), n \in \mathbb{Z}$, is compact, real analytic on $H$ and the following asymptotics estimates are fulfilled:

$$
\begin{aligned}
g_{n 1}(q) & =q_{1(c n)}+q_{2(s n)}+\ell^{d}(n), \\
\left(d_{q} g_{n 1}(q)\right)(x) & =(\cos 2 \pi n x, \sin 2 \pi n x)+\ell^{2}(n),
\end{aligned}
$$

as $n \rightarrow \infty$, uniformly on bounded subsets of $[0,1] \times B_{C}\left(p, \varepsilon_{p}\right)$ for any fixed $p \in H$.

Proof. Using Lemma 3.1 and Lemma 3.3 we obtain the needed statement.

In the following Lemma we will have the analyticity of $b_{n}^{2}$.

Lemma 3.5. Each function $b_{n}=\left|\gamma_{n}(\cdot)\right|^{2} / 4, n \in \mathbb{Z}$, is compact and real analytic on $H$. Its gradient is given by the formulas

$$
d_{q} b_{n}=-\left(z_{n}^{+}-z_{n}^{-}\right)\left[\frac{\partial \Delta\left(z_{n}^{-}, q\right)}{2 \Delta_{z}\left(z_{n}^{-}, q\right)}-\frac{\partial \Delta\left(z_{n}^{+}, q\right)}{2 \Delta_{z}\left(z_{n}^{+}, q\right)}\right], \quad z_{n}^{-} \neq z_{z}^{+},
$$




$$
d_{q} b_{n}=0, \quad z_{n}^{-}=z_{n}^{+} .
$$

Moreover,

$$
\begin{aligned}
b_{n} & =\hat{q}_{n}^{2}+\ell^{d}(n), \\
d_{q} b_{n} & =d_{q} \hat{q}_{n}^{2}+\ell^{d}(n),
\end{aligned}
$$

$n \rightarrow \infty$, uniformly on bounded subsets of $[0,1] \times B_{C}\left(p, \varepsilon_{p}\right)$ for any fixed $p \in H$.

Proof. The proof for $b_{n}$ repeats the one for $z_{n}^{0}$. To prove real analyticity, fix $p \in H$ and consider $b_{n}(q)$ for some $n \geqslant 1$. By Lemma 2.3, there exists $\varepsilon \in(0,1)$ such that for all $q \in B_{C}(p, \varepsilon)$ the function $\Delta(z, q)^{2}-1$ has exactly two roots in each disc $U_{n}^{-}(p) \subset U_{n}^{+}(p)$. We have the identity

$$
b_{n}=\frac{1}{4 \pi i} \int_{\beta_{n}(p)}\left(z-z_{n}^{0}(q)\right)^{2} F(z, q) d z,
$$

for all $q \in B(p, \varepsilon)$. The function $F(z, q), z \in \beta_{n}(p), q \in B(p, \varepsilon)$ has analytic extension on the ball $B_{C}(p, \varepsilon)$ then $b_{n}(\cdot)$ also has analytic extension on the ball $B_{C}(p, \varepsilon)$. Hence by $(3.34)$, $b_{n}(\cdot)$ is a real analytic function on $H$.

Differentiating (3.34) we obtain

$$
d_{q} b_{n}=\frac{1}{4 \pi i} \int_{\beta_{n}} 2\left(z_{n}^{0}(q)-z\right) F(z, q) d z\left(d_{q} z_{n}^{0}\right)+\frac{1}{4 \pi i} \int_{\beta_{n}}\left(z_{n}^{0}(q)-z\right)^{2} \partial F(z, q) d z,
$$

and integrating by parts we deduce that

$$
d_{q} b_{n}=\frac{1}{2 \pi i} \int_{\beta_{n}}\left(z_{n}^{0}(q)-z\right)\left(F(z, q) d_{q} z_{n}^{0}+G(z, q)\right) d z,
$$

hence using the theorem of the residue we have (3.30-31). Note that if $z_{n}^{-}=z_{n}^{+}$then we get $\partial \Delta\left(z_{n}^{-}, q\right)=0$, (see Lemma 2.2).

Using (3.11) we obtain (3.32). To prove (3.33) fix $q \in B_{C}\left(p, \varepsilon_{p}\right.$, for some $p \in H$. Then by i) of Lemma 3.2, $\left|\widetilde{z}_{n}^{ \pm}(q)-(\pi n)\right|<1$ for all $|n|>N_{p}$, that is the distance between $\widetilde{z}_{n}^{ \pm}(q)$ and the contour $c_{n}(r), r=3 / 2$, is greater than $1 / 2$. We rewrite integral (3.36) in the form

$$
d_{q} b_{n}=\frac{1}{2 \pi i} \int_{c_{n}(r)}\left(z_{n}^{0}(q)-z\right) \Delta(z, q) \frac{\left(\Delta_{z}(z, q) d_{q} z_{n}^{0}+\partial \Delta(z, q)\right) d z}{\Delta^{2}(z, q)-1} .
$$

Using (3.24), (2.47), (2.51), we have

$d_{q} b_{n}=\frac{1}{\pi i} \int_{c_{n}(r)} \frac{(z-\pi n) \cos z}{\sin ^{2} z}\left(\sin z d_{q} z_{n}^{0}-\partial \Delta(z, q)\right)\left(1+\ell^{d}(n)\right) d z=(-1)^{n} \partial \Delta(\pi n, q)+\ell^{d}(n)$, and (2.50) implies (3.33). 


\section{Analytic isomorphism}

Recall that $f_{n}(z, q)=\left(\Delta^{2}(z, q)-1\right) / w_{n}(z, q), z \in \mathbb{C}, q \in H_{C}$. In this section Theorem 1.1 will be proved. In order to get the analyticity of $g_{n 2}$ we need additional properties of $f_{n}$.

Lemma 4.1. Each function $f_{n}\left(\mu_{n}(\cdot), \cdot\right), n \in \mathbb{Z}$, is compact, real analytic and positive on $H$. Moreover,

$$
\begin{gathered}
f_{n}\left(m_{n}(q), q\right)=1+\ell^{d}(n), \\
d_{q} f_{n}\left(m_{n}(q), q\right)=\ell^{2}(n),
\end{gathered}
$$

$n \rightarrow \infty$, uniformly on bounded subsets of $[0,1] \times B_{C}\left(p, \varepsilon_{p}\right)$ for any fixed $p \in H$.

Proof. In order to prove real analyticity, fix $p \in H$ and consider $m_{n}(q)$ for some $n \in \mathbb{Z}$. By Lemma 2.3, there exists $\varepsilon \in(0,1)$ such that for all $q \in B_{C}(p, \varepsilon)$ the function $\Delta(z, q)^{2}-1$ has exactly two roots in each disc $U_{n}^{-}(p) \subset U_{n}^{+}(p)$. Moreover, by Lemma 2.2, there exists

$\varepsilon \in(0,1)$ such that for all $q \in B_{C}(p, \varepsilon)$ we get $\mu_{n}(q) \in U_{n}^{-}(p) \subset U_{n}^{+}(p)$. We have the identity

$$
f_{n}\left(m_{n}(q), q\right)=\frac{1}{2 \pi i} \int_{\beta_{n}} \frac{f_{n}(z, q) d z}{z-m_{n}(q)}
$$

for all $q \in B(p, \varepsilon)$ and rewrite the function $w_{n}$ in the form

$$
w_{n}(z, q)=b_{n}(q)-\left(z_{n}^{0}(q)-z\right)^{2} .
$$

Then by Lemmas $2.2,3.3,3.5$, the function $f_{n}(z, q), z \in \beta_{n}(p), q \in B(p, \varepsilon)$ has analytic extension on the ball $B_{C}(p, \varepsilon)$. Therefore, by Lemma 3.1, $f_{n}\left(m_{n}(\cdot), \cdot\right)$ also has analytic extension on the ball $B_{C}(p, \varepsilon)$. Hence $f_{n}\left(m_{n}(\cdot), \cdot\right)$ is a real analytic function on $H$ and by (4.3), Lemmas $2.2,3.1,3.3,3.5, f_{n}\left(m_{n}(\cdot), \cdot\right), n \in \mathbb{Z}$, is compact and positive on $H$.

To prove (4.1) fix $q \in B_{C}\left(p, \varepsilon_{p}\right)$, for some $p \in H$. Then by i) of Lemma 3.2, $\left|\widetilde{z}_{n}^{ \pm}(q)-\pi n\right|<1$ for all $|n|>N_{p}$, that is the distance between $\widetilde{z}_{n}^{ \pm}(q)$ and the contour $c_{n}(r), r=3 / 2$, is greater than $1 / 2$. Using (2.47), (3.4), (3.32), we obtain

$$
f_{n}(z, q)=\frac{\sin ^{2} z+\ell^{d}(n)}{(z-\pi n)^{2}\left(z-m_{n}(q)\right)}, \quad z \in c_{n}(r), q \in B\left(p, \varepsilon_{p}\right) .
$$

Substituting (4.5) into (4.3), with $c_{n}=\beta_{n}$, we get

$$
f_{n}\left(m_{n}(q), q\right)=\frac{1}{2 \pi i} \int_{c_{n}} \frac{\left(\sin ^{2} z+\ell^{d}(n)\right) d z}{(z-\pi n)^{2}\left(z-m_{n}\right)}=\frac{1}{2 \pi i} \int_{c_{n}} \frac{\left(\sin ^{2} z+\ell^{d}(n)\right) d z}{(z-\pi n)^{3}}=1+\ell^{d}(n)
$$

Differentiating (4.3), with $c_{n}=\beta_{n}$, we have

$$
d_{q} f_{n}\left(m_{n}(q), q\right)=\frac{1}{2 \pi i} \int_{c_{n}} \frac{f_{n}(z, q) d z}{\left(z-m_{n}(q)\right)^{2}} d_{q} m_{n}(q)+\frac{1}{2 \pi i} \int_{c_{n}} \frac{\partial f_{n}(z, q) d z}{z-m_{n}(q)}
$$

Asimptotic estimates (4.5), (3.4) give

$$
\frac{1}{2 \pi i} \int_{c_{n}} \frac{f_{n}(z, q) d z}{\left(z-m_{n}(q)\right)^{2}}=\frac{1}{2 \pi i} \int_{c_{n}} \frac{\left(\sin ^{2} z+\ell^{d}(n)\right) d z}{(z-\pi n)^{2}\left(z-m_{n}(q)\right)^{2}}=\ell^{2}(n)
$$


In order to estimate the second term in (4.6) we write $\partial f_{n}$ in the form

$$
\partial f_{n}(z, q)=\frac{1}{w_{n}(z, q)}\left\{-f_{n}(z, q) \partial w_{n}(z, q)+\partial \Delta(z, q)^{2}\right\}, \quad z \in c_{n}(r) .
$$

Identity $(4,4)$ and (3.24-25) yield

$$
\partial w_{n}(z, q)=d_{q} b_{n}+2\left(z-z_{n}^{0}\right) d_{q} z_{n}^{0}=d_{q} q_{n}^{2}+\ell^{2}(n)=\ell^{2}(n), \quad z \in c_{n}(r) .
$$

Then (4.7), (3.32), (3.24) imply

$$
\partial f_{n}(z, q)=-\frac{\partial \Delta(z, q)^{2}+\ell^{2}(n)}{(z-\pi n)^{2}}, \quad z \in c_{n}(r)
$$

Using these estimates and (3.4), (2.47), (2.48-50) we obtain

$$
\int_{c_{n}} \frac{\partial f_{n}(z, q) d z}{z-m_{n}(q)}=-\int_{c_{n}} \frac{\partial \Delta(z, q)^{2}+\ell^{2}(n)}{(z-\pi n)^{3}}=-\int_{c_{n}} \frac{\cos z \partial \Delta(z, q)+\ell^{2}(n)}{(z-\pi n)^{3}}=\ell^{2}(n) .
$$

Below we need the properties of the mapping $h_{n 2}(\cdot)$ from [K9].

Lemma 4.2. Each function $h_{n 2}(\cdot), n \in \mathbb{Z}$, is compact and real analytic on $H$. Its gradient is given by the formula

$$
d_{q} h_{n 2}=\frac{(-1)^{n}}{\sinh h_{n 2}}\left(\partial \Delta\left(m_{n}(q), q\right)+\Delta_{z}\left(m_{n}(q), q\right) d_{q} m_{n}\right), \quad h_{n 2} \neq 0
$$

Moreover,

$$
\begin{aligned}
h_{n 2}(q) & =-q_{1(s n)}+q_{2(c n)}+\ell^{d}(n), \quad n \rightarrow \infty, \\
\left(d_{q} h_{n 2}(q)\right)(x) & =(-\sin 2 \pi n x, \cos 2 \pi n x)+\ell^{2}(n), \quad n \rightarrow \infty,
\end{aligned}
$$

uniformly on bounded subsets of $[0,1] \times B_{C}\left(p, \varepsilon_{p}\right)$ for any fixed $p \in H$.

Note that by Lemma 4.1 , each function $\sqrt{f_{n}\left(m_{n}(\cdot), \cdot\right)}$ is compact, real analytic, positive on $H$.

Lemma 4.3. Each function $g_{n 2}(\cdot), n \in \mathbb{Z}$, is compact, real analytic on $H$ and the identity is fulfilled:

$$
g_{n 2}(q)=\frac{1}{\sqrt{f_{n}\left(m_{n}(q), q\right)}} \sinh h_{n 2}(q) .
$$

Moreover,

$$
\begin{gathered}
g_{n 2}=-q_{1(s n)}+q_{2(c n)}+\ell^{d}(n), \quad n \rightarrow \infty, \\
d_{q} g_{n 2}=(-\sin 2 \pi n x, \cos 2 \pi n x)+\ell^{2}(n), \quad n \rightarrow \infty,
\end{gathered}
$$

uniformly on bounded subsets of $[0,1] \times B_{C}\left(p, \varepsilon_{p}\right)$ for any fixed $p \in H$.

Proof. For any $q \in H$ the following identity is fulfilled:

$$
\sinh ^{2} h_{n 2}=\Delta^{2}\left(m_{n}(q), q\right)-1=f_{n}\left(m_{n}(q), q\right) g_{n 2}^{2}(q),
$$


Recall that since $f_{n}>0$, we deduce that the function $1 / \sqrt{f_{n}}$ has an analytic extension in some ball $B_{C}(q, \varepsilon)$ for some $\varepsilon>0$ and since $h_{n 2}(\cdot)$ is real analytic we get (4.11). Using Lemmas 4.1-2 and (4.11) we derive that $g_{n 2}(\cdot)$ is compact and real analytic on $H$ and estimates (4.12-13) are fulfilled.

To check ii) of Theorem A we recall the well known Paley-Wiener result ( see [Le]).

Lemma 4.4. Suppose that $f(z)$ is an entire function with

$$
\sup _{z \in \mathbb{C}}\left|f(z) e^{-|z|}\right|<\infty, \quad \quad \int_{\mathbb{R}}|f(x)|^{2} d x<\infty
$$

and $f\left(z_{n}\right)=0$ for some sequence $\left\{z_{n}\right\}_{-\infty}^{\infty}$ of distinct real numbers such that $z_{n}=\pi n+o(1)$ as $|n| \rightarrow \infty$. Then $f \equiv 0$.

Now we prove our main theorem.

Proof of Theorem 1.1. We check all conditions of Theorem A for the map $g: H \rightarrow \ell^{2} \oplus \ell^{2}$.

i) By Lemmas 3.4, 4.3, each function $g_{n}(\cdot), n \in \mathbb{Z}$, is compact, real analytic on $H$, and estimates (3.28), (4.12) are fulfilled. Due to (3.28), (4.12) the mapping $g(q)$ is locally bounded; using the uniform boundedness principle, we deduce that $g(q)$ is real analytic.

ii) It follows from $(3.29),(4.13)$ that $d_{q} g-\Phi$ is a compact operator for all $q \in H$; so, $d_{q} g$ is a Fredholm operator. Assume that $y \in H$ is a solution of the equation

$$
\left(d_{q} g\right) y=0, \text { or }\left\{\left(d_{q} g_{n}, y\right)=0, n \in \mathbb{Z}\right\}, \quad y \neq 0,
$$

for some $q \in H$. Introduce the function

$$
f(z)=\int_{0}^{1}(\partial \Delta)(x, z, q) y(x) d x, \quad z \in \mathbb{C} .
$$

First, assume $g_{n}=0$, then Lemma 2.2 yields $f\left(z_{n}\right)=0$, for $z_{n}^{0}=z_{n}^{ \pm}$.

Second, let $g_{n} \neq 0$, then using (4.15) we get $\left(d_{q} b_{n}, y\right)=0$, and identity (2.30) implies

$$
-\frac{f\left(z_{n}^{-}\right)}{\Delta_{z}\left(z_{n}^{-}\right)}+\frac{f\left(z_{n}^{+}\right)}{\Delta_{z}\left(z_{n}^{+}\right)}=0 .
$$

Also, $\Delta_{z}\left(z_{n}^{+}\right) \Delta_{z}\left(z_{n}^{-}\right)<0$. Hence $f\left(z_{n}^{-}\right) f\left(z_{n}^{+}\right) \leqslant 0$ and there exists a point $z_{n} \in\left[z_{n}^{-}, z_{n}^{+}\right]$such that $f\left(z_{n}\right)=0$. Thus, there exists $z_{n}=\pi n+o(1)$ as $n \rightarrow \infty$. In order to show $f \in L^{2}(\mathbb{R})$ we use (2.48-49) which imply

$$
\begin{gathered}
f(z)=-\frac{1}{2} \int_{0}^{1} \int_{0}^{1}\left(\left[q_{1}(x+t) \cos z(2 t-1)+q_{2}(x+t) \sin z(2 t-1)\right] y_{1}(t)+\right. \\
\left.\left(q_{2}(x+t) \sin z(2 t-1)+q_{1}(x+t) \cos z(2 t-1)\right) y_{2}(t)\right) d t d y+\ell^{d}(n)
\end{gathered}
$$

and then

$$
\left.f(z)=\int_{0}^{1}\left[G_{1}(t) \cos z(2 t-1)+G_{2}(t) \sin z(2 t-1)\right] d t+\ell^{d}(n)\right)
$$




$$
G_{p}(y)=\int_{0}^{1} q_{p}(y+t)\left(y_{1}(t)+y_{2}(t)\right) d t, \quad p=1,2, \quad|z-\pi n| \leqslant \pi, \quad|n| \rightarrow \infty, \quad d>1,
$$

and note $G_{1}, G_{2} \in L(\mathbb{R})$. Hence $f$ is the Fourier transform of the functions $G_{1}, G_{2}$ plus the function from $L^{2}(\mathbb{R})$. Hence by Lemma $4.4, f \equiv 0$.

Using $f(z) \equiv 0$ and (3.23) we obtain $\left(d_{q} z_{n}^{0}, y\right)=0$. Therefore, $\left(d_{q} g_{n 1}, y\right)=-\left(d_{q} \mu_{n}, y\right)=0$ for all $n \in \mathbb{Z}$. Suppose that $\left(d_{q} h_{n 2}, y\right)=0$ for all $n \in \mathbb{Z}$. Note that for any fixed $q \in H$ the vectors $\left\{\left(d h_{n 2}\right),\left(d m_{n}\right), n \in \mathbb{Z}\right\}$ form a basis of $H$ (see [K9]). Then $y=0$ and the operator $d_{q} g(q)$ is invertible.

In order to show $\left(d_{q} h_{n 2}, y\right)=0$ we consider two cases. First, let $h_{n 2} \neq 0$. Then by (4.8), $\left(d_{q} h_{n 2}, y\right)=0$. Second, let $h_{n 2}=0$. Then by (4.11), $g_{n 2}=0$ and using Lemma 4.3 we have $\left(d_{q} g_{n 2}, y\right)=\left(d_{q} h_{n 2}, y\right)=0$.

iii) Estimates (1.3) were proved in [K3].

iv) By Lemmas 3.4, 4.3, each mapping $g_{n}: H \rightarrow \mathbb{R}^{2}, n \in \mathbb{Z}$, is compact.

v) By (5.16), the needed set is compact, since $Q_{2}$ is the Hamiltinian.

Therefore, all conditions of Theorem A are fulfilled and then $g$ is the real analytic isomorphism between $H, \ell^{2} \oplus \ell^{2}$.

\section{$5 \quad$ Identities and Estimates}

We consider a conformal mapping on the complex plane with parallel slits. We call the domain $\mathcal{K}=\mathbb{C} \backslash \cup{ }_{n}$ the "comb" where the slit , ${ }_{n}=\left[u_{n}-i h_{n}, u_{n}+i h_{n}\right]$ with the height $h_{n} \geqslant 0, n \in \mathbb{Z}$. Here $u_{n+1}-u_{n}>0$ and $u_{n} \rightarrow \pm \infty$ as $n \rightarrow \pm \infty$. Let the domain $\mathcal{Z}=\mathbb{C} \backslash \cup \bar{\gamma}_{n}$ where the non-overlapping slits $\gamma_{n}=\left(z_{n}^{-}, z_{n}^{+}\right) \subset \mathbb{R}$ with the length $\left|\gamma_{n}\right|=z_{n}^{+}-z_{n}^{-} \geqslant 0, n \in \mathbb{Z}$. Here $z_{n}^{+}<z_{n+1}^{-} \leqslant z_{n+1}^{+}$and $z_{n}^{+} \rightarrow \pm \infty$ as $n \rightarrow \pm \infty$. We shall study a conformal mapping $k(z)$ from $\mathcal{Z}$ onto some comb $\mathcal{K}$ with the conditions $k(i v)=i y+\frac{Q_{0}+o(1)}{i y}$ as $v \rightarrow \infty$, where $k=u+i v$ and let $z=x+i y$. It is well known that $k(z)$ is a continuous function in $z \in \overline{\mathcal{Z}}$ (see [L]). Let $z(k)$ be the inverse function for $k(z)$. In this case we introduce the sets $\sigma_{n} \equiv\left[z_{n-1}^{+}, z_{n}^{-}\right]=z\left(\left[u_{n-1}, u_{n}\right]\right), n \in \mathbb{Z}$. We call $\sigma=\cup \sigma_{n}$, the spectrum of the corresponding conformal mapping $z, \gamma_{n}$ a gap in the spectrum. If $\gamma_{n}$ is empty, then the components $\sigma_{n}, \sigma_{n+1}$ merge. It is well known that the set $\sigma$ can not be the spectrum of two different conformal mappings (see $[\mathrm{L}]$ ).

Lemma 5.1. Let the conformal mapping $k(\cdot)$ satisfy the condition $\sum\left|\gamma_{n}\right|<\infty$. Then

$$
|u(x)-x| \leqslant \sum\left|\gamma_{n}\right|, \quad x \in \mathbb{R}
$$

Proof. The function $x-u(x)$ is increasing only on the gaps $\gamma_{n}, n \in \mathbb{Z}$. Moreover, $x-u(x) \rightarrow 0$ as $x \rightarrow \pm \infty$ (see [KK2]). Then $\sup |u(x)-x| \leqslant \sum\left|\gamma_{n}\right|$.

Below we need the simple estimates from [KK2]

$$
\begin{gathered}
\frac{\left|\gamma_{n}\right|^{2}}{8} \leqslant A_{n} \leqslant \frac{1}{\pi} h_{n}\left|\gamma_{n}\right|, \\
\left|\gamma_{n}\right| \leqslant 2 h_{n},
\end{gathered}
$$


Define the functionals $Q_{m}, P_{m}, U, S$, as in the case $u_{n}=\pi n, n \in \mathbb{Z}$. We now prove some identities. Recall $h_{+}=\sup h_{n}, \beta=\max \left\{1, \frac{h_{+}}{\pi}\right\}$.

Theorem 5.2. Assume that $\|h\|_{1}<\infty$. Then $Q_{2}<\infty$ and the following identities are fulfilled:

$$
\begin{gathered}
\frac{1}{\pi} \iint\left|\left(k^{2}(z)-z^{2}\right)^{\prime}\right|^{2} d x d y=8 S, \\
Q_{1}=\frac{1}{\pi} \int x v(x) d x=\frac{1}{\pi} \int u(x) v(x) d x=P_{1}, \\
Q_{2}=S+\frac{Q_{0}^{2}}{2}=P_{2}-\frac{1}{3} U+Q_{0}^{2}, \\
S+\frac{1}{3} U=P_{2}+\frac{Q_{0}^{2}}{2} .
\end{gathered}
$$

Proof. Using the estimate from [KK2] $Q_{2} \leqslant \sum \rho_{n}^{2} h_{n}^{2}, \quad \rho_{n}=\max \left|z_{n}^{ \pm}\right|$, and the inequality $\rho_{n} \leqslant C+C_{1}\left(1+h_{+}\right)|\pi n|, n \in \mathbb{Z}$, for some constants $C, C_{1}$, from [K3], we obtain

$$
Q_{2} \leqslant \sum\left(C+C_{1}\left(1+h_{+}\right)|\pi n|\right)^{2} h_{n}^{2}<\infty .
$$

Let $k^{2}(z)-z^{2}=\phi(z)+i f(z), \phi=\left(u^{2}(z)-v^{2}(z)-x^{2}+y^{2}\right), f=2(u(z) v(z)-x y)$. Using the Green formula we obtain

$$
\begin{gathered}
\iint_{\mathbb{C}_{+}}\left|\left(k^{2}(z)-z^{2}\right)^{\prime}\right|^{2} d x d y=\iint_{\mathbb{C}_{+}}|\nabla f(z)|^{2} d x d y=-\int f(x) f_{y}(x) d x \\
=-\int 2 u(x) v(x) \phi^{\prime}(x) d x=\int 4 u(x) v(x)\left(v(x) v^{\prime}(x)+x\right) d x=4 \int x u(x) v(x) d x,
\end{gathered}
$$

which yields (5.4). First we show (5.5-6) for the finite gap case. In this case we have

$$
k(z)=z-\frac{Q_{0}}{z}-\frac{Q_{1}}{z^{2}}-\frac{Q_{2}}{z^{3}} \ldots, \quad|z| \rightarrow \infty .
$$

(see $[\mathrm{KK} 2])$. Due to (5.8) we have for large $t$ :

$$
Q_{1}=\frac{1}{2 \pi i} \int_{|z|=t} k(z)(k(z)-z) d z
$$

and since $k(z)(k(z)-z)=\left(u^{2}-v^{2}-u v\right)+i v(2 u-x), x \in \mathbb{R}$, we get

$$
Q_{1}=\frac{1}{2 \pi i} \int_{|z|=t}(u+i v)(u+i v-x-i y) d z=\frac{1}{2 \pi} \int(2 u v-x v) d x
$$

then $Q_{1}=2 P_{1}-Q_{1}$ which yields (5.5).

Using (5.8) we have

$$
Q_{2}-Q_{0}^{2}=\frac{1}{2 \pi i} \int_{|z|=t} z k(z)(k(z)-z) d z
$$


and since $\left.z k(z)(k(z)-z)=x\left(u^{2}-v^{2}-x u\right)\right)+i x v(2 u-x), x \in \mathbb{R}$ we obtain

$$
Q_{2}-Q_{0}^{2}=\frac{1}{2 \pi} \int x v(2 u-x) d x=2 S-Q_{2}
$$

which yields the first identity in (5.6). Using (5.8) again we deduce that

$$
Q_{2}-2 Q_{0}^{2}=\frac{1}{2 \pi i} \int_{|z|=t} k^{2}(z)(k(z)-z) d z
$$

and since $k^{2}(z)(k(z)-z)=\left(u^{3}-3 u v^{2}-x u^{2}+x v^{2}\right)+i v\left(3 u^{2}-v^{3}-2 x u\right), x \in \mathbb{R}$ we have

$$
Q_{2}-2 Q_{0}^{2}=\frac{1}{2 \pi} \int v\left(3 u^{2}-v^{3}-2 x u\right) d x=3 P_{2}-U-2 S,
$$

and the identity $2 Q_{2}=2 S+Q_{0}^{2}$ implies the second one in (5.6).

Combining identities in (5.6) we obtain (5.7).

Introduce the set $\sigma^{r}=\sigma \cup(-\infty, r) \cup(r, \infty)$, the variables and the functionals corresponding $\sigma^{r}$ denote by upper index $r$. It is well known (see [Le]) that $v^{r}(x) \nearrow v(x), u^{r}(x) \rightarrow u(x)$ as $r \rightarrow \infty, \quad x \in \mathbb{R}$. Using this, estimate $\left|u^{r}(x)-x\right| \leqslant \sum\left|\gamma_{n}\right|$ and Lebegues's theorem we have the convergence $Q_{m}^{r} \rightarrow Q_{m}, \quad P_{m}^{r} \rightarrow P_{m}, \quad m=1,2, S^{r} \rightarrow S, \quad U^{r} \rightarrow U$, as $r \rightarrow \infty$.

We prove Theorem 1.3 with more exact constants.

Lemma 5.3. Let $\|h\|_{1}<\infty$. Then the following identities are fulfilled:

$$
\begin{gathered}
h_{+}^{2} \leqslant 2 Q_{0} \leqslant 2 \sqrt{2 Q_{2}}, \\
Q_{0} \leqslant \frac{2}{\pi^{2}}\left(\left|\gamma_{0}\right|+\|\gamma\|_{1}\right)^{2}, \\
Q_{2} \leqslant \frac{1}{\pi 2}\|h\|_{1}^{2}+\frac{4}{\pi^{2}}\|h\|^{4}, \quad\|h\|_{1}^{2} \leqslant(2 \pi)^{3} \beta^{2} Q_{2}, \quad \beta=\max \left\{1, \frac{h_{+}}{\pi}\right\}, \\
\frac{1}{4}\|J\|_{1}^{2}+\frac{1}{3}\|J\|^{4} \leqslant Q_{2}=\frac{1}{4}\|J\|_{1}^{2}+\|J\|^{4}, \\
\frac{1}{32}\|\gamma\|^{2}+\frac{1}{192}\|\gamma\|_{1}^{2} \leqslant Q_{2} \leqslant \pi \beta^{2}\|\gamma\|_{1}^{2}+4 Q_{0}^{2},
\end{gathered}
$$

Proof. The first inequality in (5.12) was proved in [KK3], see [K5] also. The second one in (5.12) follows from (5.6).

Let $d=\sum\left|\gamma_{n}\right|$, then $d \leqslant\left|\gamma_{0}\right|+\|\gamma\|_{1}(1 / 2 \sqrt{3})$ since $\sum n^{-2}=\pi^{2} / 6$. Using the simple estimate $Q_{0} \leqslant h_{+} d / \pi$, (see [KK1]) we obtain $h_{+}^{2} \leqslant 2 Q_{0}$ we have $Q_{0} \leqslant\left(2 / \pi^{2}\right) d^{2} \leqslant\left|\gamma_{0}\right|^{2}+\|\gamma\|_{1}^{2}$. The identity $Q_{0}=\|J\|^{2}$ and estimate (5.3) yield $h_{+}^{2} \leqslant 2 Q_{0}=2\|J\|^{2}$.

Using identity (5.2-3) and (5.6) we obtain

$$
Q_{2} \leqslant P_{2}+Q_{0}^{2} \leqslant \frac{1}{4 \pi}\|h\|_{1}\|g\|_{1}+\frac{4}{\pi^{2}}\|h\|^{4} \leqslant \frac{1}{2 \pi}\|h\|_{1}^{2}+\frac{4}{\pi^{2}}\|h\|^{4}
$$

We need some result from [K3]. Suppose that a real function $f$ belongs to the Sobolev space $W_{1}^{2}(D(t))$, where a domain $D(t)=\{0<u<\pi, 0<v<t\}$ for some $t>0$. Let $f$ obey 
the following conditions : $f(u+i 0)=0, u \in(0, \pi)$, and $f$ is continuous in $\bar{D}(t)$. Then the following estimate is fulfilled:

$$
\int_{0}^{t} \frac{|f(+0+i v)|^{2} d v}{v} \leqslant \frac{\pi}{2} \max \left\{1, \frac{t}{\pi}\right\} \iint_{D(t)}|\nabla f(k)|^{2} d u d v, \quad k=u+i v .
$$

We take $f=\operatorname{Im}\left(k^{2}-z^{2}\right)=2(u v-x y)$, the domain $D_{n}=(\pi(n-1), \pi n] \times(0, \infty)$, and by (5.17),

$$
\int_{0}^{h_{n}} \frac{\left(2 v u_{n}\right)^{2}}{v} d v=2\left(u_{n} h_{n}\right)^{2} \leqslant \frac{\pi^{2}}{2} \beta I_{n}, \quad I_{n}=\frac{1}{\pi} \iint_{D_{n}}|\nabla f|^{2} d u d v,
$$

Then using (5.4) we obtain

$$
\|h\|_{1}^{2}=\sum(2 \pi n)^{2} h_{n}^{2} \leqslant \sum \pi^{2} \beta I_{n}=(2 \pi)^{2} \beta S .
$$

Using the inequalities

$$
S^{2} \leqslant Q_{2} P_{2}, \quad P_{2} \leqslant \frac{1}{2 \pi}\|h\|_{1}^{2},
$$

we have

$$
\|h\|_{1}^{2} \leqslant 4 \pi^{2} \beta Q_{2}^{1 / 2} \frac{1}{\sqrt{2 \pi}}\|h\|_{1},
$$

which implies the second estimate in (5.14).

Inequality (5.12) implies

$$
U \leqslant \frac{h_{+}^{2}}{\pi} \int v(x) d x \leqslant 2 Q_{0}^{2}
$$

and using (5.6) we obtain

$$
Q_{2} \geqslant P_{2}+\frac{1}{3} Q_{0}^{2}=\frac{1}{4}\|J\|_{1}^{2}+\frac{1}{3}\|J\|^{4} .
$$

Again using (5.6) we get $Q_{2} \leqslant P_{2}+Q_{p}^{2}$ which implies (5.15).

Using the first inequality in (5.21) and (5.2) we have

$$
Q_{2} \geqslant P_{2}+\frac{1}{3} Q_{0}^{2} \geqslant \frac{1}{32}\|\gamma\|_{1}^{2}+\frac{1}{192}\|\gamma\|^{4} .
$$

Substituting (5.2) into (5.6) we have

$$
Q_{2} \leqslant P_{2}+Q_{0}^{2} \leqslant \frac{1}{4 \pi}\|\gamma\|_{1}(2 \pi)^{3 / 2} \beta \sqrt{Q_{2}}+Q_{0} \sqrt{2 Q_{2}}
$$

which implies (5.16).

Acknowledgments. The author is grateful for financial support given by SFB 288 and for the hospitality of the KTH (Stockholm).

\section{References}

[BGGK] D. Battig, B. Grebert, J. Guillot, T. Kappeler: Foliation of phase space for the cubic non-linear Schrödinger equation, Composition Math., 1993, 85, 163-199. 
[DT] Deift P., Trubowitz E.: Inverse scattering on the line, Commun. Pure and Applied Math. 32, 121-251, 1979

[FM] Flashka H., McLaughlin D. Canonically conjugate variables for the Korteveg- de Vries equation and the Toda lattice with periodic boundary conditions. Prog. of Theor. Phys. 55, (1976), 438-456.

[Fa] Faddeev L.: Properties of the $S$-matrix of the one-dimensional Schrödinger equation Trudy Mat. Inst. Steklov 73, 1964, 314-333 (Russian)

[F] Firsova N. : Riemann surface of a quasimomentum, and scattering theory for a perturbed Hill operator, (Russian) Mathematical question in the theory of wave propagation, 7. Zap. Nauchn. Sem. Leningrad Otdel. Mat. Inst. Steklov (LOMI) 51, 183-196 (1975), English transl. in J. Soviet Math. 11, 487-497 (1979)

[F1] Firsova, N.: The direct and inverse scattering problems for the one dimensional perturbed Hill operator. Math. USSR Sbornik 58, 351-388 (1987)

[GT1] Garnett J., Trubowitz E.: Gaps and bands of one dimensional periodic Schrödinger operators. Comment. Math. Helv. 59, 258-312 (1984)

[GT2] Garnett, J., Trubowitz, E.: Gaps and bands of one dimensional periodic Schrödinger operators II. Comment. Math. Helv. 62, 18-37 (1987)

[Ka] T. Kappeler: Fibration of the phase space for the Korteveg-de-Vries equation. Ann. Inst. Fourier (Grenoble), 41, 1, 539-575 (1991).

[KK1] Kargaev P., Korotyaev E.: The inverse problem for the Hill operator, the direct approach. Invent. Math. 129, no 3, 567-593 (1997) Erratum, Invent. Math.138, 1999, 227

[KK2] Kargaev P., Korotyaev E.: Effective masses and conformal mappings. Commun. Math. Phys. 169, 597-625 (1995).

[K1] Korotyaev E.: The inverse problem and the trace formula for the Hill operator, II. Math. Z. 231, 1999, 345-368

[K2] E. Korotyaev: The inverse problem for the Hill operator I. Internat. Math. Res. Notices 1997, 3, 113-125.

[K3] Korotyaev E. : Metric properties of conformal mappings on the complex plane with parallel slits. Internat. Math. Res. Notices 1996, 10, 493-503.

[K4] Korotyaev E. : The propagation of the waves in the one-dimensional periodic media. Asymptotic Analysis, 15, 1-24, (1997)

[K5] Korotyaev E.: The estimate of periodic potentials in terms of effective masses. Commun. Math. Phys. 1997, 183, 383-400

[K6] Korotyaev E. Estimate for the Hill operator.I, J. Diff. Eq. 162, 1-26, 2000

[K7] Korotyaev E.: Estimate of periodic potentials in terms of gap lengths. Commun. Math. Phys. 1998, 197, no. 3, 521-526

[K8] Korotyaev E. : Periodic weighted operator. preprint SFB-288, No. 388,1999

[K9] Korotyaev E.: Marchenko-Ostrovski mapping for periodic Zakharov-Shabat systems, preprint SFB-288, 1999, will be published in J. Diff. Eq.

[K10] Korotyaev E. : Inverse problem for periodic weighted operator, J. Func. Anal. 170, $188-218,2000$

[Kr] Krein M. : On the characteristic function $A(\lambda)$ of a linear canonical system differential equation of the second order with periodic coefficients (Russian), Prikl. Mat. Meh. 21, 
320-329 (1957)

[L] Levin B.: Majorants in the class of subharmonic functions.1-3. Theory of functions, functional analysis and their applications. 51, 3-17(1989); 52, 3-33 (1989). Russian.

[Le] Levinson N. Gap and density theorem. Amer. Math. Soc. Coll. Publ., New York, 1940 [LS] Levitan B., Sargsjan I. Sturm-Liouville and Dirac operators. Translated from the Russian. Mathematics and its Applications (Soviet Series), 59. Kluwer Academic Publishers Group, Dordericht, 1991, 350 pp.

[MO1] Marchenko V., Ostrovski I.: A characterization of the spectrum of the Hill operator. Math. USSR Sb. 26, 493-554 (1975).

[MO2] Marchenko V., Ostrovski I.: Approximation of periodic by finite-zone potentials. Selecta Math. Sovietica. 1987, 6, No 2, 101-136.

[Mi1] Misyura T. Properties of the spectra of periodic and antiperiodic boundary value problems generated by Dirac operators. I,II, Theor. Funktsii Funktsional. Anal. i Prilozhen, (Russian), 1978, 30, 90-101; 1979, 31, 102-109

[Mi2] Misyura T. Finite-zone Dirac operators. Theor. Funktsii Funktsional. Anal. i Prilozhen, (Russian), 1980, 33, 107-11

[PT] Pöschel, P., Trubowitz E.: Inverse Spectral Theory. Boston: Academic Press, 1987.

[T] Titchmarsh E.: The theory of functions. Sec. ed., Univ. Press, London, 1975 